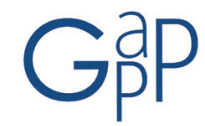

GESTIÓN Y ANÁLISIS DE POLÍTICAS PÚBLICAS, Nueva Época, nº 12 julio-diciembre 2014 ISSN: 1989-8991

DOI: http://dx.doi.org/10.24965/gapp.voi12.10204

\title{
Potencialidad y limitaciones del modelo de diferencias en diferencias aplicado con datos agregados a la evaluación de políticas públicas en el ámbito territorial'
}

\author{
Julian Pérez García \\ Profesor de Econometría de la Universidad Autónoma de Madrid. \\ julian.perez@uam.es \\ Francisco Perez Hernández \\ Profesor de Economía Financiera de la Universidad Autónoma de Madrid.
}

f.perez@uam.es

Recibido: 19 de abril de 2014

Aceptado: 19 de julio 2014

\begin{abstract}
Resumen
Elobjetivo del presente artículo es la ilustración de la potencialidad del Modelo de Diferencias en Diferencias (DiD) para la evaluación de diversas políticas públicas aplicadas en el ámbito territorial y utilizando datos agregados. Con esta finalidad se ha realizado una panorámica general sobre las alternativas de evaluación de políticas públicas y revisado las posibilidades de aplicación sobre un amplio espectro de políticas desarrolladas en la comunidad autónoma del País Vasco. A continuación, se presenta la metodología básica de los modelos de diferencias en diferencias y se realizarán dos aplicaciones alternativas, una de ámbito local, el Programa lzartu, y otra de ámbito regional, el Programa Euskadi de fomento de la sociedad de la información con el fin de ilustrar la potencialidad y limitaciones de la metodología propuestas. El artículo finaliza con un apartado dedicado a la valoración de los resultados obtenidos y con una propuesta para la corrección de los principales problemas metodológicos identificados vinculados con el cumplimiento de la condición de tendencia paralela.
\end{abstract}

Palabras clave

Políticas públicas, evaluación de programas, modelo de diferencias en diferencias.

Clasificación JEL: $\mathrm{H}_{53}, 138,038, \mathrm{R} 13, \mathrm{R} 58$

\section{Potentiality and limitations of the Differences-in-Differences Model when it's applied to aggregate data to evaluate public policies territorially}

\footnotetext{
Abstract

The aim of this paper is the illustration of the potential of the Differences in Differences Model (DiD) for the evaluation of various public policies in the territory and using aggregate data. For this purpose, has made an overview on alternative evaluation of public policies and reviewing the implementation possibilities of a wide range of policies implemented in the País Vasco. Herein are included, the basic methodology of the differences in differences models and two alternative applications, one being local, Programa Izartu and the other regional, Programa Euskadi to promote the information society in order to illustrate the potential and limitations of the methodology proposed. The article concludes with a section on the assessment of the results and a proposal for correction of identified major methodological problems associated with the fulfillment of the condition of parallel trend.

Key words

Public policies, program evaluation, differences in differences model.

JEL Classification: $\mathrm{H}_{53}, \mathrm{I38}, \mathrm{O} 38, \mathrm{R} 13, \mathrm{R} 58$

$1 \quad$ El presente artículo resume los resultados obtenidos en el desarrollo de un programa de investigación más amplio denominado Proyecto DEPURE realizado por la Dirección General de Economía y Planificación del Gobierno Vasco en el contexto de la Convocatoria Europea INTERREG IIIC, a quien los autores agradecen la financiación y colaboración aportada. En cualquier caso, las opiniones y valoraciones contenidas en el mismo son responsabilidad exclusiva de los autores y no deben ser consideradas como conclusiones oficiales del proyecto.
} 


\section{INTRODUCCIÓN}

Durante los últimos años, el análisis de los efectos de la implantación de políticas públicas ha sido objeto de un número significativo de investigaciones. Si bien es cierto que los estudios sobre la evaluación de políticas han proliferado sustancialmente, el análisis del impacto de los programas destinados a la implantación de cualquier política es complejo y poco concluyente, ya que los conceptos que rodean a dicha temática son heterogéneos y dan origen a aproximaciones desarrolladas por la teoría de la administración y gestión pública o por diversos métodos cuantitativos (Görg y Strobl, 2005; Planas, 2005; Douglas, 2006).

Debido a la heterogeneidad que existe en las aproximaciones disponibles en la literatura sobre evaluación de políticas públicas, nosotros consideramos algunas características generales sobre este proceso en función del objetivo perseguido con la implantación de las políticas públicas en un determinado entorno.

Así, podemos identificar dos grupos de políticas. El primero, de aplicación directa, se basa en que los resultados obtenidos pueden ser directamente constatados u observados sin ningún tipo de instrumento adicional de medición o valoración; y el segundo, de modificación del entorno socioeconómico, que requiere de una reacción posterior del resto de agentes sociales (García Gómez, 2005).

En este artículo nos centramos en el segundo grupo, es decir, en aquellas políticas que persiguen una modificación del entorno socioeconómico y cuyo cambio debería ser contrastado una vez implementa la política pública que se desea evaluar.

Lógicamente, para poder realizar adecuadamente este tipo de análisis es necesario disponer de la información adecuada y que se resume, básicamente, en la medición de los indicadores de respuesta adecuados y de algún tipo de medición "contrafactual" que nos permita identificar cuál habría sido ese nivel de respuesta de los agentes si no se hubiera aplicado dicha política pública.

Así, para contrastar los efectos finales de determinadas políticas de modificación del entorno socioeconómico consideramos que la alternativa metodológica basada en los Modelos de Diferencias en Diferencias (DiD) es la técnica econométrica que mejor se adapta a nuestro objetivo, principalmente porque podríamos establecer diferencias en el comportamiento medio de dos grupos o colectivos que han experimentado un determinado tratamiento, en nuestro caso, la aplicación de una determinada política pública, tratando de filtrar los posibles efectos de otros factores ajenos a dicho tratamiento y que en principio afectan de forma similar a ambos colectivos (Görg y Strobl, 2005; Douglas, 2006; Banzhaf y Walsh, 2006).

Ahora bien, a la hora de poner en práctica estos procesos de evaluación el principal problema con el que nos enfrentamos es la carencia de información dado que en muy pocas ocasiones se dispone de los indicadores o mediciones específicas del comportamiento previo y las respuestas posteriores de los grupos de tratamiento (aquellos donde se ha aplicado la política concreta a evaluar) y los de control (donde no se han aplicado), lo que ha limitado la utilización real de esta metodología.

Frente a estas circunstancias, el objetivo central de nuestro artículo consiste en la ilustración de la aplicabilidad de este tipo de modelos sobre datos agregados, disponibles, generalmente, en los diferentes institutos y centros de estadística, y que, por tanto, no precisan de ser generados "ad hoc" para la política que se pretende evaluar.

Para abordar esta ilustración se presentan dos aplicaciones concretas realizadas respectivamente sobre políticas de ámbito local y políticas de ámbito regional, más concretamente, el Programa Izartu de desarrollo local y el Programa Euskadi de fomento de la Sociedad de la Información (S.I.), reiterando que nuestro objetivo no se centra en la evaluación concreta de estos programas sino en la aplicabilidad y validez de la metodología de los modelos de diferencias en diferencias, tratando de delimitar sus posibles carencias cuando se aplican sobre este tipo de datos agregados y proponiendo algunas mejoras metodológicas para salvar los principales problemas detectados.

El artículo está estructurado de la siguiente forma. En el apartado 2 se realiza una revisión general y sin ánimo de exhaustividad, de los conceptos que engloban la evaluación de políticas públicas. En el apartado 3 se describe la metodología empírica básica de los modelos de diferencia en diferencias, mientras que en el cuarto se presentan los dos ejemplos de aplicación seleccionados. El apartado 5 se destina a la presentación de las mejoras metodológicas propuestas para la corrección de los problemas detectados y se finaliza con un último apartado dedicado al resumen y conclusiones obtenidas. 


\section{LA EVALUACIÓN DE POLÍTICAS PÚBLICAS}

El concepto etimológico que rodea a los mecanismos de evaluación de políticas públicas es complejo e inconcluso, ya que, tanto la definición de evaluación, como el de políticas públicas, presentan una elevada heterogeneidad y dan origen a multitud de aproximaciones, tanto desde la teoría de la administración y gestión pública, como desde la propia economía o los métodos cuantitativos².

Debido a la heterogeneidad que existe en las aproximaciones disponibles en la literatura sobre evaluación de políticas públicas, es necesario considerar, a priori, las características generales de las políticas a evaluar y determinar claramente el objetivo perseguido en este proceso de evaluación.

Así, podemos identificar un primer grupo de políticas, que podemos denominar de aplicación directa, y donde los resultados obtenidos pueden ser directamente constatados u observados sin ningún tipo de instrumento adicional de medición o valoración. Este es el caso de todo tipo de infraestructuras o servicios públicos, beneficios o prestaciones sociales, realización de estudios, análisis, proyectos, etc. (Planas, 2005).

Por otro lado, existen un conjunto de actuaciones, o políticas públicas, que persiguen un objetivo adicional de modificación del entorno socioeconómico y que precisan, por tanto, de una reacción posterior del resto de agentes sociales (familias, empresas, organizaciones, etc.). Ejemplos de este segundo grupo, son los Planes de fomento de la empresarialidad, formación, desarrollo de la sociedad de la información, fomento de la I+D+i, etc. (García-Gómez, 2005)

Es importante destacar que los procesos de evaluación del primer tipo de políticas pasarían por la constatación directa de la ejecución de la misma dentro de los plazos y condiciones establecidas a priori, mientras que en el segundo grupo sería necesario realizar un análisis posterior de las modificaciones, o cambios reales, inducidos sobre el entorno socioeconómico.

En cuanto a los procesos de evaluación, podemos diferenciar, al menos, tres niveles de valoración de políticas:

1. Cumplimiento o ejecución, que consiste en la constatación de la ejecución de la política propuesta de acuerdo con los objetivos prefijados (temporales y económicos). En esta primera etapa del proceso de evaluación bastaría con disponer de los programas de implantación y/o presupuestos ejecución y la información sobre los resultados u objetivos alcanzados por lo que, en principio, no precisa del desarrollo de indicadores y/o modelos específicos de valoración. A su vez, dentro de la clasificación propuesta por Planas (2005), podemos distinguir tres tipos de técnicas:

a. de auditoría, que hacen referencia a la comprobación de la veracidad en el cumplimiento de objetivos sin cuestionar su validez, dimensión o adecuación;

b. de control de gestión, que consiste en el análisis de las correspondencia entre los planes de gobierno y las acciones realizadas y;

c. de presupuesto por programas, que analiza la relación entre el uso de recursos y los objetivos presupuestados.

2. Intensidad de uso o impacto directo. Este nivel de evaluación consiste en la cuantificación de los efectos directos de la política implementada y requiere de la especificación de indicadores cuantitativos concretos que pueden ser analizados de forma directa o comparativa con otras políticas similares. Según Planas (2005), los procedimientos incluidos en este segundo grupo son:

a. presupuesto por resultados, que se refiere a la construcción de indicadores para el seguimiento de las actividades del gobierno y la verificación del cumplimiento de políticas;

b. gerencialismo público, utilización generalizada de indicadores de gestión.

3. Efectos inducidos sobre el entorno socioeconómico. Por último, el tercer nivel es considerado el más complejo, ya que requiere de un desarrollo metodológico específico para su cuantificación, siendo su objetivo último la identificación de los valores de respuesta inducidos sobre el resto de agentes como resultado de la implantación de la política concreta. Para la realización de este tipo de evaluaciones es necesario disponer de una batería de información suficientemente representativa, tanto de la actuación de los agentes sociales, como del entorno socioeconómico que se pretende modificar. En este tercer grupo

2 Véase, por ejemplo, el monográfico de Ekonomiaz, del tercer cuatrimestre del 2005, dedicado a la problemática de la evaluación de políticas públicas. 
se incluirían el conjunto de técnicas denominadas de evaluación de programas y donde tiene la aplicación metodológica que se analiza en este artículo, el Modelo de Diferencias en Diferencias (DiD).

A efectos de ilustrar las diferentes tipologías de políticas públicas, así como los distintos niveles de evaluación se ha elaborado la siguiente tabla donde se recogen, por filas los diferentes niveles de evaluación y, por columnas, la tipología de políticas.

TABLA 1

POLÍTICAS Y NIVELES DE EVALUACIÓN

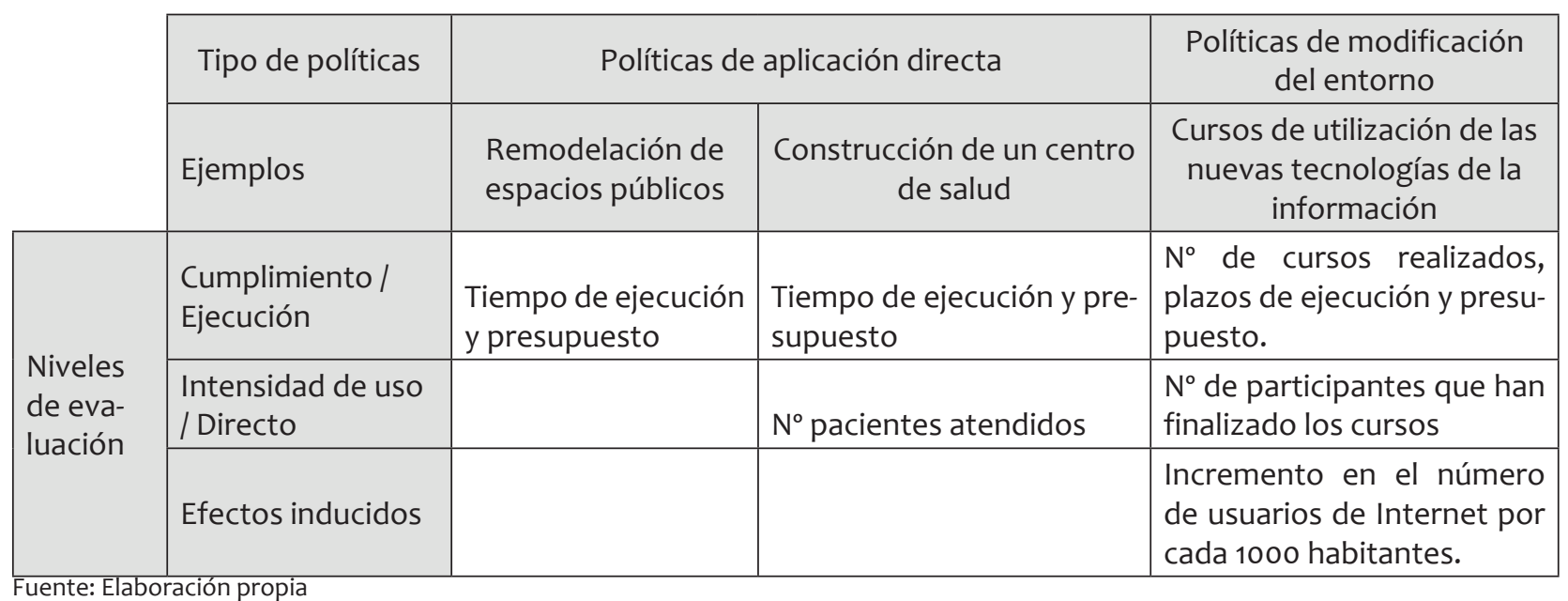

La tabla 1 recoge algunos ejemplos propuestos. El primero de ellos es la remodelación de espacios públicos, como pueden ser la creación y construcción de nuevos parques públicos. La evaluación fundamental consistiría en la comparación entre los objetivos programados y los efectivamente ejecutados tanto en términos de tiempos como presupuestarios, así como la constatación de los niveles de calidad de la obra finalmente recepcionada.

En el segundo caso, la construcción y puesta en marcha de un nuevo centro de salud, el nivel de evaluación se iniciaría con un análisis similar al anterior en cuanto a cumplimientos y ejecución de la obra presupuestada y, posteriormente, se podría realizar un segundo análisis de los efectos directos o intensidad de uso, utilizando indicadores cuantitativos del tipo, número de pacientes atendidos, tiempos de atención, etc. Este segundo nivel de evaluación puede realizarse de forma directa, es decir, recogiendo y procesando la información cuantitativa sobre los indicadores seleccionados, o bien utilizando procedimientos más complejos de comparación con otros centros similares mediante técnicas de posicionamiento (benchmarking) o de análisis de eficiencia (Planas, 2005).

El tercer ejemplo propuesto corresponde a una política de modificación del entorno socioeconómico cuyo objetivo último es la potenciación del uso de las nuevas Tecnologías de la Información y la Comunicación (TIC), o el desarrollo de la denominada Sociedad de la Información y en el que la acción pública se concreta en la oferta de cursos de formación en nuevas tecnologías (por ejemplo el uso de Internet).

En este caso, los dos primeros niveles de evaluación son similares a los casos anteriores y se centran en la valoración de la actividad pública desarrollada, es decir, los propios cursos de formación. Así, en el primer nivel se verifica la celebración de los cursos programados de acuerdo a los objetivos temporales y presupuestarios establecidos, mientras que en el segundo nivel se realiza un análisis del efecto directo o intensidad de uso de los mismos, utilizando indicadores del tipo del número de participantes que han finalizado los cursos, niveles de destreza alcanzados por los participantes, etc.

Sin embargo, y dado que el objetivo perseguido en este artículo consiste en el fomento del uso de las TIC, es necesario contrastar, a posteriori, si el hecho de haber realizado estas políticas ha tenido una respuesta adecuada por parte de los ciudadanos y si se han visto modificados los niveles de uso globales de dichas TIC, aumentando, por ejemplo, el número de usuarios de Internet por cada 1000 habitantes, el nivel de equipamiento informático de los hogares o el número de transacciones on line realizadas.

Lógicamente, para poder realizar adecuadamente este tipo de análisis es necesario disponer de la información adecuada y que se resume, básicamente, en la medición de los indicadores de respuesta adecuados y de algún 
tipo de medición "contrafactual” que nos permita identificar cuál habría sido ese nivel de respuesta de los agentes si no se hubiera aplicado dicha política pública.

En la práctica este tipo de información puede recogerse de forma temporal, es decir, midiendo los niveles de respuesta antes y después de la ejecución de la política concreta, o bien utilizando los denominados grupos de control, que serían colectivos o individuos a los que no se ha aplicado la política concreta.

Así, para analizar este planteamiento, proponemos la utilización de los modelos de diferencias en diferencias, técnica econométrica que se ajusta mejor a este tipo de planteamientos cuantitativos, ya que utilizan ambos tipos de información.

Es importante destacar que los ejemplos utilizados deben interpretarse como orientativos y no necesariamente normativos o excluyentes, ya que, por ejemplo, la política de remodelación de espacios públicos, puede ser evaluada mediante indicadores de efectos directos o intensidad de uso, (número de visitantes diarios que recibe un determinado parque), y la implantación de un nuevo centro de salud puede ser objeto de una evaluación de sus efectos inducidos sobre el entorno socioeconómico (mediante indicadores de niveles de salud de la población de referencia).

De la misma forma, no todas las políticas de modificación del entorno pueden ser susceptibles de valorar sus efectos indirectos, bien porque su objetivo final sea difícilmente cuantificable en términos de indicadores medibles (nivel de satisfacción general de los ciudadanos, o democratización y transparencia de las instituciones), o bien porque no sea posible el establecimiento de las situaciones contrafactuales. Es decir, cuál habría sido el nivel de respuesta de los agentes en ausencia de dicha política.

En los siguientes apartados, se realiza una propuesta metodológica para analizar la evaluación de políticas públicas a nivel local y regional para el caso español. Primero se identificarán un conjunto de políticas públicas y posteriormente, aplicáremos un modelo de diferencias en diferencias a nivel local y regional con el objetivo de detectar fuerzas y debilidades sobre la implantación de las mismas.

\section{METODOLOGÍA DE ESTIMACIÓN}

Tal como se establecía en la introducción, el objetivo de este artículo es poner de manifiesto la potencialidad y, fundamentalmente, las debilidades, o problemas, que presenta la metodología de los modelos de diferencias en diferencias para su aplicación operativa en la evaluación de determinadas políticas públicas utilizando datos agregados.

Los modelos de diferencias en diferencias, o estimador de diferencias en diferencias, es una técnica econométrica que se basa en la determinación estadística de las diferencias en el comportamiento medio de dos grupos o colectivos, uno de los cuales ha experimentado un determinado tratamiento, en nuestro caso, la aplicación de una determinada política pública, tratando de filtrar los posibles efectos de otros factores ajenos a dicho tratamiento y que, en principio, afectan de forma similar a ambos colectivos.

De forma sencilla, los modelos DiD establecen niveles medios de respuesta de estos dos colectivos, denominados respectivamente grupo de tratamiento y grupo de control, antes y después de recibir el tratamiento, o aplicación de cualquier proceso de política pública que se pretenda analizar, asumiendo que las diferencias entre los niveles de respuesta del grupo de tratamiento, una vez recibido el mismo, frente al grupo de control, se deben exclusivamente a dicho tratamiento pudiendo identificarse, por tanto, como el efecto neto del mismo.

Si bien este tipo de análisis experimentales o quasi-experimentales tienen aplicación en otras disciplinas como la medicina o la psicología, las aplicaciones al campo económico son relativamente recientes, ya que, aunque existen algunos trabajos previos, las primeras aplicaciones relevantes se empezaron a publicar a mediados de la década de los noventa3; si bien es cierto que en los últimos años han proliferado los estudios que utilizan esta metodología para analizar los efectos de multitud de políticas y actuaciones públicas, la aplicación de esta metodología es heterogénea y, por ejemplo, se han realizado aplicaciones a diversas tipologías de políticas públicas, tales como la educación y la formación, Duflo (2001), Görg y Strobl (2005) y Shapiro y Skoufias (2006); políticas

3 En el campo de la economía laboral destacan la aplicaciones recogidas por Heckman, Lalande y Smith (1999) sobre los programas de formación y empleo. Impacto de la inmigración sobre los salarios y del salario mínimo sobre el empleo, Card (1990) y Card y Krueger (1993). Efectos del salario mínimo en la formación, Neumark y Wascher (2001) y sobre políticas activas de empleo, Blundell et al. (2001). En la economía de la salud y en el análisis del denominado Estado del Bienestar también ha sido objeto de múltiples aplicaciones de esta metodología pudiendo destacarse las realizadas por Gruber y Madrian (1994), Hamermesh y Scoones (1999), Rub (2003), Song (2003), Ten Cate (2003), Domino et al.(2004), Konetzka et al. (2004), Acs y Nelson (2004), Liu, Dow y Norton (2004), Hoynes, Gelbach y Bitter (2005) y Shen y Zuckerman (2005). 
sobre impuestos, Eissa y Liebman (1996), Blundell, Duncan y Meghir (1998) y Hansson (2008); y medioambiente, Eissa y Liebman(1996), Blundell, Duncan y Meghir (1998), Douglas(2006) y Banzhaf y Walsh (2006).

Para el caso español, la aplicación de esta metodología sobre la evaluación de políticas públicas es escasa. Nosotros hemos identificado dos trabajos de impacto a nivel local y regional de España. En Planas (2005) se desarrolla un análisis conceptual sobre aplicación de políticas públicas en España y García (2005) realiza una evaluación del Programa de Atención Dental Público (PADI) en el País Vasco.

En su especificación más simple, el modelo de diferencias en diferencias se plantearía mediante una expresión como la siguiente:

$$
Y_{i j, t}=\alpha+\beta * G_{j}+\gamma^{*} D_{t}+\delta * T_{j}+\varepsilon_{j, t}
$$

donde:

$$
\begin{aligned}
& G_{j}=\left\{\begin{array}{ccc}
1 & \dot{\boldsymbol{s}} & j \in \text { Grupo } d \text { tratamiento } \\
0 & \dot{\boldsymbol{s}} & j \in \text { Grupo } \boldsymbol{d} \text { control }
\end{array}\right. \\
& D_{t}=\left\{\begin{array}{lll}
1 & \dot{\boldsymbol{s}} & t \geq \text { punto } \boldsymbol{d} \text { tratamiento } \\
0 & \dot{\boldsymbol{s}} & t<\text { punto } \boldsymbol{d} \text { tratamiento }
\end{array}\right. \\
& T_{j t}=\left\{\begin{array}{lll}
1 & \dot{\boldsymbol{s}} & D_{t}=1 y G_{j}=1 \\
0 & \boldsymbol{a} & \text { caso contrario }
\end{array}\right.
\end{aligned}
$$

La variable endógena $Y_{i j, t}$ recoge el nivel de respuesta en cada momento del tiempo $t$, de un individuo concreto $i$, incluido en un grupo $j$, con respecto al objetivo concreto sobre el que se pretende realizar la evaluación de la política pública implantada. $G_{j}, D_{t}$ y $T_{t j}$ son variables discretas que toman valores o ó 1, donde $G_{j}$ determina la pertenencia de cada individuo al grupo de tratamiento, es decir los que han recibido la política a analizar,

$(\mathrm{G}=1)$ o al grupo de control $(\mathrm{G}=0), D_{t}$ determina el momento temporal en que se ha realizado el tratamiento (por ejemplo, la aplicación de la política), tomando valor cero para los periodos previos a la implantación de la política pública $(D=0)$ y uno para los periodos posteriores $(D=1)$ y $T_{j}$ identifica las observaciones de los individuos que pertenecen al grupo de tratamiento una vez recibido este. $\varepsilon_{i j, t}$ es el término del error o perturbación aleatoria

En [1], el coeficiente $\alpha$, recoge el nivel de respuesta medio de los individuos incluidos en el grupo de control, es decir, aquellos sobre los que no se aplicará la política a analizar antes de recibir el tratamiento.

El coeficiente $\beta$ recoge la diferencia media que presentan el conjunto de individuos incluidos en el grupo de tratamiento, aquellos a los que se aplicará la política, respecto al grupo de control, en los periodos previos a la aplicación de la política en análisis. Es decir, mientras los individuos incluidos en el grupo de control presentan un nivel medio de respuesta en la variable objetivo $Y_{i j, t}$ equivalente al valor de $\alpha$ en los periodos anteriores a la aplicación de la política, los individuos incluidos en el grupo de tratamiento presentarán un nivel medio de respuesta igual a $(\alpha+\beta)$ en ese mismo periodo.

$$
\alpha=\frac{1}{I_{G=0} * N_{D=0}} \sum_{G=0, D=0} Y_{j, t}=\bar{Y}_{G=0, D=0}
$$




$$
(\alpha+\beta)=\frac{1}{I_{G=1} * N_{D=0}} \sum_{G=1, D=0} Y_{j, t}=\bar{Y}_{G=1, D=0}
$$

El coeficiente $\gamma$ representa la evolución media experimentada en el nivel de respuesta de los individuos incluidos en el grupo de control durante los periodos posteriores a la aplicación de la política, y recoge, por tanto, todos aquellos factores que han influido sobre dichos niveles de respuesta a lo largo del tiempo y que no pueden ser asimilados al efecto de dicha política.

Por tanto la suma de los coeficientes $(\alpha+\gamma)$ recoge el valor medio de respuesta de los individuos incluidos en el grupo de control en los periodos posteriores a la aplicación de la política a analizar.

$$
(\alpha+\gamma)=\frac{1}{I_{G=0} * N_{D=1}} \sum_{G=0, D=1} Y_{j, t}=\bar{Y}_{G=0, D=1}
$$

Finalmente, el coeficiente $\delta$ recoge el efecto real de la política aplicada sobre la variable de respuesta escogida. Este es nuestro coeficiente de interés, ya que representa la diferencia media en los valores de respuesta del grupo de tratamiento frente al grupo de control, una vez aplicada la política a analizar, ya que el resto de factores que podrían estar afectando a dichos niveles de respuesta han sido "filtrados" por el coeficiente $\gamma$.

De esta forma el nivel medio de respuesta que presentarían el conjunto de individuos incluidos en el grupo de tratamiento, con posterioridad a la aplicación de éste, vendría definido por la suma de los cuatro coeficientes del modelo $\alpha+\beta+\gamma+\delta$ (Smith y Todd, 2005a; Girma y Görg, 2007).

$$
(\alpha+\beta+\gamma+\delta)=\frac{1}{I_{G=1} * N_{D=1}} \sum_{G=1, D=1} Y_{j, t}=\bar{Y}_{G=1, D=1}
$$

El método de estimación de [1] puede ser a través de Mínimos Cuadrados Ordinarios (MCO) o bien utilizando una especificación de Datos de Panel, asumiendo las hipótesis habituales. De esta forma, no sólo podríamos calcular el efecto neto de la política analizada sino que podríamos matizar su nivel de significatividad estadística. Sin embargo, las características propias de las variables utilizadas suelen inducir incumplimientos sistemáticos de estas hipótesis de partida por lo que la validación estadística habitual puede verse seriamente afectada. Esto es debido, principalmente, a dos motivos: inexistencia de matrices escalares de varianzas y covarianzas de la perturbación aleatoria, y la existencia de heterocedasticidad, o presencia de autocorrelación (Bertrand et al., 2004).

En estos casos si bien los estimadores calculados siguen manteniendo, en general, buenas propiedades estadísticas, los contrastes de significatividad individual de parámetros se ven sesgados, bien por infravaloración o por sobrevaloración de las desviaciones típicas.

Aunque en la literatura especializada se han planteado soluciones alternativas a estos problemas, en la práctica habitual no son demasiado usuales, tal como señalan Bertrand et al. (2004) y Puhani (2012).

Desde una óptica alternativa a la estimación econométrica clásica, el coeficiente de interés de [1], $\delta$, puede obtenerse a partir de los valores medios de respuesta presentados por los distintos grupos, antes y después de recibir el tratamiento, utilizando una expresión del tipo:

$$
\hat{\delta}=\left(\bar{Y}_{G=1, D=1}-\bar{Y}_{G=1, D=0}\right)-\left(\bar{Y}_{G=0, D=1}-\bar{Y}_{G=0, D=0}\right)
$$

definiendo los distintos valores medios $\bar{Y}$ tal como se recoge en las expresiones [2] a [5]. 
Como puede comprobarse fácilmente el efecto de la política aplicada puede obtenerse por diferencia entre los valores medios que presenta el grupo de tratamiento antes y después de haberlo recibido menos las diferencia que presenta el grupo de control en ese mismo periodo.

Este modelo de diferencias en diferencias así planteado, es una potente herramienta de evaluación de todo tipo de políticas públicas aplicadas, tanto en el ámbito local como en el regional. Sin embargo debemos ser conscientes de las necesidades y limitaciones que puede presentar a la hora de realizar los correspondientes análisis empíricos.

Así, para poder aplicar correctamente el modelo propuesto es necesario disponer de una base de datos homogénea, que contenga información de la variable objetivo antes y después de realizar el tratamiento (aplicación de la política que se pretende evaluar) y tanto en individuos a los que se le ha aplicado como a los que no.

En términos de políticas locales supondría disponer de información homogénea de un conjunto de municipios donde se haya aplicado la política concreta y otros donde no se haya aplicado; tanto antes como después de aplicar dicha política. Para el caso de políticas regionales sería necesario disponer de datos homogéneos tanto de la región donde se ha aplicado la política, como de otras regiones en donde no se hayan implementado políticas similares.

Por otra parte y al margen del cumplimiento de las condiciones de identificación (tendencia paralela, y estabilidad en la composición), debemos ser conscientes de las limitaciones metodológicas que presenta el método propuesto y relacionadas con aspectos tales como:

1. Existencia de diferentes "grados" de aplicación de la política a analizar.

En el planteamiento básico se asume que la política en cuestión se implementa de forma completa y homogénea en todos los individuos incluidos en el grupo de tratamiento, sin embargo en muchas ocasiones las políticas tienen múltiples niveles o grados de intensidad que pueden dificultar la adecuada cuantificación del efecto.

2. Existencia de posibles desfases entre la implantación de la política y la aparición de los efectos buscados.

De la misma forma el modelo asume que la implantación de la política se produce en un momento temporal concreto a partir del cual empieza a generar efectos completos sobre la variable de respuesta, mientras que son bastante habituales las políticas de implantación progresiva que se introducen a lo largo de varios periodos o que tienen procesos de maduración (periodo que transcurre entre la implantación y la generación de respuestas) más largos.

3. Dificultad de medición de la variable objetivo.

Teniendo en cuenta que los efectos de la política a analizar se determinan en función de una variable de respuesta concreta es necesario disponer de una adecuada medición de dicho nivel de respuesta; sin embargo, tampoco es infrecuente la existencia de políticas con objetivos múltiples, que no se pueden recoger en una única variable o, simplemente, que no es una magnitud medible.

4. Concurrencia de otros factores no contemplados

Finalmente, no debemos olvidar que todo el análisis se basa en los supuestos de permanencia del resto de condiciones que determinan el entorno en el que se registran los distintos niveles de repuesta (Asunción de la hipótesis ceteris paribus) y que pueden concurrir otra serie de factores, no contemplados en el modelo y que puedan distorsionar los resultados obtenidos.

\section{APLICACIÓN DEL MODELO DE DIFERENCIAS EN DIFERENCIAS EN LA EVALUACIÓN DE POLÍTICAS EN EL PAÍS VASCO}

Antes de realizar el planteamiento empírico ilustrativo de la utilización del modelo de diferencias en diferencias para la evaluación de políticas públicas locales y regionales es importante poner de manifiesto algunos factores que condicionan significativamente las posibilidades de aplicación de dicha metodología.

Por un lado nos encontramos con que una buena parte de las políticas implementadas constituyen programas complejos que incorporan un gran número de acciones diferenciadas que, a efectos de la metodología propuesta, deberían ser analizadas de forma individual, ya que tienen, tanto objetivos, como destinatarios, diferentes. 
Adicionalmente, esta misma complejidad de objetivos haría necesaria la utilización, en muchos casos, de distintas variables de respuesta con el fin de identificar correctamente los efectos inducidos sobre el entorno socioeconómico.

Por otra parte, la existencia de programas de larga duración temporal, o de renovación anual, dificulta nuevamente la aplicación de la metodología propuesta, ya que los posibles efectos se irían solapando en el tiempo y no podrían ser claramente identificados.

Para realizar la selección adecuada para la aplicación empírica se analizaron un conjunto de políticas públicas locales y regionales implementadas en la Comunidad Autónoma del País Vasco y que aparecen recogidas en un listado inicial de 84 políticas elaborado en el año 2007 por el Sistema Vigía de Desarrollo Regional (SVDR).

Como punto de partida se diferenciaron dos grandes grupos de políticas en función del ámbito básico de aplicación de las mismas. De la muestra inicial de 84 políticas iniciales se identificaron 41 políticas locales y 43 se ubicaron en el ámbito regional. Posteriormente, y con el objetivo de depurar la muestra inicial, se realizó una clasificación de las 84 políticas públicas implantadas en el País Vasco teniendo en cuenta la tipología recogida y expuesta en el anexo 1 de este trabajo.

Así, se diferenciaron las que considerábamos de aplicación directa y que, inicialmente, no podrían ser susceptibles de evaluación mediante la metodología propuesta, de aquellas que tenían un objetivo último de modificación del entorno. Es importante señalar que, esta clasificación es puramente orientativa ya que la complejidad de muchas de las políticas identificadas dificulta su clasificación sin un estudio pormenorizado de las mismas, análisis que no es el objetivo de este artículo.

Del conjunto de 41 políticas de actuación local identificadas la mitad podrían ser incluidas dentro del grupo de actuación directa y que serían susceptibles, a priori, de ser evaluadas mediante la metodología propuesta.

Dentro de este grupo, al menos 14 de las 20 políticas de acción directa identificadas en el ámbito local podrían ser valoradas mediante indicadores de intensidad, o uso directo y, adicionalmente, 7 de estas 14 podrían ser también evaluadas desde un punto de vista de los efectos inducidos sobre el entorno socioeconómico, es decir que se podríamos utilizarlas en la metodología propuesta en este artículo como variables de respuesta.

Finalmente, para la ilustración de la aplicabilidad en el caso de políticas locales, se seleccionó el Programa Izartu cuya primera fase se ejecutó entre los años 2001 y 2005. La selección de este programa para realizar la aplicación está vinculada con la disponibilidad de información estadística, así como por la relevancia del mismo dentro del conjunto de actuaciones locales en el País Vasco.

Por su parte, para el conjunto de políticas se seleccionó el Plan Euskadi de fomento de la Sociedad de la Información ya que, además de ser uno de los programas que ya ha finalizado, presenta objetivos delimitados y cuantificables. Por otra parte, al igual que sucede en el caso de políticas locales, la disponibilidad de información estadística de este programa fue un factor determinante para la elección de ésta, además la información es adecuada tanto en la dimensión temporal como en la regional. ${ }^{4}$

\section{1.- Aplicación a políticas Locales: El programa Izartu}

El Programa Izartu es un instrumento dirigido a la revitalización socioeconómica y la regeneración territorial de zonas urbanas desfavorecidas y deficitarias del País Vasco. Con esta finalidad, esta iniciativa consiste en la cofinanciación de programas integrales ejecutados a nivel local, programas que abordan de forma global todos los problemas específicos de la zona. De esta forma el Programa Integral de Revitalización Urbana de la Comunidad Autónoma del País Vasco responde a la necesidad de complementar las políticas sectoriales de cohesión social con actuaciones y medidas más integrales para cada una de las áreas urbanas degradadas.

El fin último del programa radica en la búsqueda de una mayor vertebración y cohesión territorial, económica y social de la zona, estableciéndose como objetivos básicos la regeneración de zonas urbanas degradadas, el alcance de una mayor cohesión territorial, social y económica, adaptación de un esquema similar al de la política regional de la Unión Europea y la financiación de actuaciones de carácter integral complementarias a las que realizan los departamentos de Gobierno y otorgando un mayor protagonismo a los ayuntamientos 5 .

De acuerdo con la información recogida en el primer informe de seguimiento del programa Izartu, publicado en Julio del 2002, del total de los 250 municipios integrados en el País Vasco, 48 habían presentado, al menos, un programa, tal como se recoge en el siguiente gráfico.

4 Para un mayor detalle sobre la identificación de políticas públicas véase el anexo 1 de este artículo.

5 En el Anexo 2 de este artículo se detallan los ejes básicos y líneas de actuación del Programa Izartu. 
FIGURA 1

MUNICIPIOS DEL PAÍS VASCO CON PROGRAMAS IZARTU

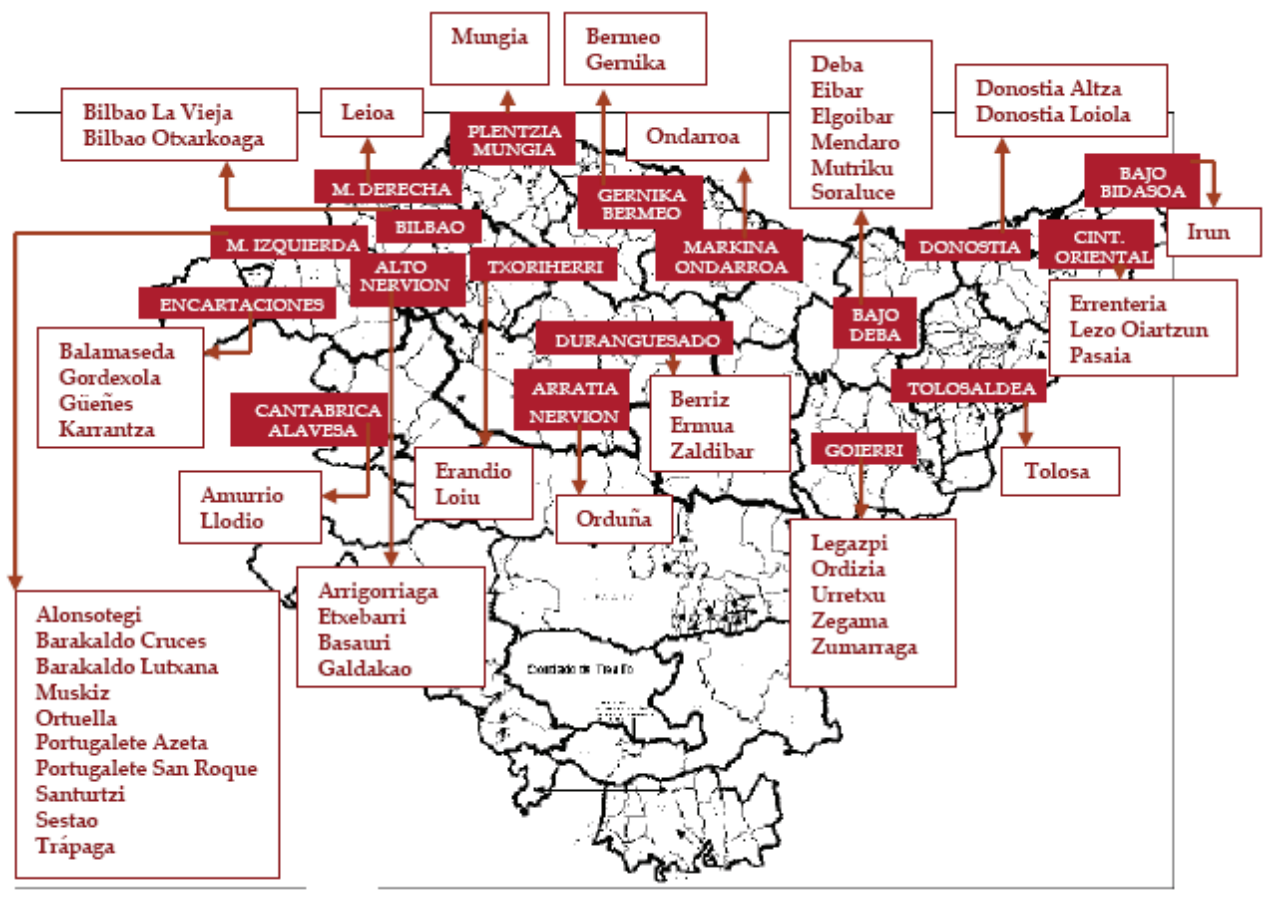

Fuente: Programa Izartu. Informe de seguimiento 2001. Julio 2002.

A la vista de estos datos podemos deducir fácilmente que estamos ante un proyecto muy complejo y que presenta múltiples dimensiones. Partiendo de esta multidimensionalidad de objetivos realizaremos la aproximación a la evaluación del programa Izartu utilizando distintas variables de respuesta que se aproximen de la mejor manera posible a los mismos.

Teniendo en cuenta que las distintas acciones se ejecutan a nivel municipal, es necesario disponer de una base de datos con información desagregada a dicho nivel y que presente datos anteriores y posteriores a la ejecución del programa, y que hemos establecido en el ejercicio del 2001.

El Instituto Vasco de Estadística (EUSTAT) ofrece una amplia batería de 206 indicadores municipales agrupados en torno a 28 categorías $^{6}$ y que contienen información desde que se dispone de datos hasta el año 2005 , si bien es cierto que no todas las series están disponibles para todos los ejercicios ya que las fuentes originales, fundamentalmente los censos, no se realizan anualmente.

De forma complementaria, hemos utilizado la información estadística del Anuario Económico de La Caixa, elaborado por el Instituto L.R. Klein de la Universidad Autónoma de Madrid?.

Una vez analizada la información disponible y los distintos objetivos del Programa Izartu se seleccionaron las siguientes variables de respuesta (indicadores) agrupadas en cinco categorías básicas:

6 Territorio, Población, Movimiento Natural de la Población, Población residente por lugar de nacimiento, Movimientos migratorios, Familias según tamaño y tipo, Sistema educativo: alumnos..., Población de 10 y más años, según nivel de instrucción, Población de 2 y más años, según nivel global de euskera, Lengua materna, Lengua hablada en casa, Edificios, según destino del edificio, Establecimientos, Viviendas, Viviendas principales, según sus instalaciones y servicios(\%), Población según relación con la actividad, Población ocupada según ramas de actividad, Movilidad de la población ocupada y estudiante según tipo, Centros extra hospitalarios públicos, Número de centros de servicios sociales, Número de oficinas bancarias, Parque de vehículos, Liquidación Presupuestaria del Gasto del Ayuntamiento (Miles de $€$ ), Renta personal y familiar, Estructura económica. Distribución del VABsf (\%), Distribución de la superficie agrícola(Ha.), Unidades ganaderas, Parque de maquinaria agrícola

7 Ofrece series de indicadores municipales homogéneos que se extienden desde mediados de los años 90 hasta la actualidad: Población residente, Tasa de paro registrado, Cuota de Mercado, Líneas telefónicas (Telefónica), Total vehículos de motor, Automóviles, Camiones y furgonetas, Otros vehículos de motor, Total entidades de crédito, Bancos, Cajas de ahorro, Cooperativas de crédito, Total licencias del comercio al por menor, Superficie de centros comerciales. 
TABLA 2

SELECCIÓN DE INDICADORES PARA VALORAR EL PROGRAMA IZARTU

\begin{tabular}{|c|c|c|}
\hline Categorías & Variables de respuesta & Definición \\
\hline $\begin{array}{l}\text { Infraestructu- } \\
\text { ras } \\
(\mathrm{M} 1)\end{array}$ & $\begin{array}{l}N^{\circ} \text { de Centros de servi- } \\
\text { cios sociales (CPO). }\end{array}$ & $\begin{array}{l}\text { Número de centros servicios sociales por cada } 1000 \text { habitantes. In- } \\
\text { cluyen asociaciones, servicios técnicos generales, centros de orienta- } \\
\text { ción, centros de día, residencias y otros. }\end{array}$ \\
\hline \multirow{2}{*}{$\begin{array}{l}\text { Actividad Eco- } \\
\text { nómica } \\
(\mathrm{M} 2)\end{array}$} & $\begin{array}{l}\mathrm{N}^{0} \text { Total de estableci- } \\
\text { mientos productivos } \\
(\mathrm{EST}) .\end{array}$ & $\begin{array}{l}\text { Número total de establecimientos productivos ubicados en cada mu- } \\
\text { nicipio por cada } 1000 \text { habitantes. Incluyen industria y energía, cons- } \\
\text { trucción, comercio, hostelería y transportes, banca, seguros y servi- } \\
\text { cios a empresa otras actividades de servicios. }\end{array}$ \\
\hline & Renta bruta (RPC). & $\begin{array}{l}\text { Es el resultado de partir el valor estimado de renta calculado por Eus- } \\
\text { tat en } 2001 \text { para cada municipio y se ha extrapolado se ha extrapola- } \\
\text { do para los años anteriores y posteriores en función de las tasas de } \\
\text { crecimiento de la renta disponible per capita estimadas en base a los } \\
\text { datos del Anuario Económico de la Caixa. }\end{array}$ \\
\hline \multirow[t]{2}{*}{$\begin{array}{l}\text { Cohesión } \\
\left(\mathrm{M}_{3}\right)\end{array}$} & $\begin{array}{l}\text { Índice de renta relativo } \\
\text { (IRE). }\end{array}$ & $\begin{array}{l}\text { Se ha calculado a partir de los datos totales de renta disponible na- } \\
\text { cional publicados por el INE y las cuotas de mercado publicadas en el } \\
\text { Anuario Económico de la Caixa, Posteriormente, se calcula la renta } \\
\text { disponible per cápita dividiendo dichos valores por la población de } \\
\text { los municipios y el total de la Comunidad del País Vasco Finalmente, } \\
\text { se obtiene el índice relativo de renta dividiendo los valores específi- } \\
\text { cos de cada municipio por el total de la comunidad en cada uno de } \\
\text { los años. }\end{array}$ \\
\hline & Tasa de paro (TPA). & $\begin{array}{l}\text { Es el cociente entre el paro registrado y el total de la población. Te- } \\
\text { niendo en cuenta que no se dispone de una adecuada medición de } \\
\text { la población activa municipal, es importante destacar que no puede } \\
\text { compararse cuantitativamente con los valores habituales de tasa de } \\
\text { paro. }\end{array}$ \\
\hline $\begin{array}{l}\text { Demografía } \\
\left(\mathrm{M}_{4}\right)\end{array}$ & $\begin{array}{l}\text { Saldo migratorio neto } \\
\text { (SMI). }\end{array}$ & $\begin{array}{l}\text { Es el saldo migratorio neto por cada } 1000 \text { habitantes está calculado } \\
\text { por cociente entre la diferencia entre las inmigraciones recibidas por } \\
\text { cada municipio menos las emigraciones registradas dividido entre la } \\
\text { población total. }\end{array}$ \\
\hline \multirow{2}{*}{$\begin{array}{l}\text { Entidades loca- } \\
\text { les } \\
\text { (M5) }\end{array}$} & $\begin{array}{l}\text { Gastos totales de los } \\
\text { ayuntamientos (GTO). }\end{array}$ & $\begin{array}{l}\text { Es la suma de los gastos corrientes y gastos de capital liquidados por } \\
\text { cada ayuntamiento divido por la población total. }\end{array}$ \\
\hline & $\begin{array}{l}\text { Gastos de capital de los } \\
\text { ayuntamientos (GCAP). }\end{array}$ & $\begin{array}{l}\text { Es el cociente entre los gastos de capital de cada ayuntamiento y el } \\
\text { total de la población. }\end{array}$ \\
\hline
\end{tabular}

El horizonte temporal de todas las variables es de 1997-2004, excepto en el caso de la tasa de paro donde no se disponía de información para el año 1997 y se tomó referencia el periodo 1998-2005. De esta forma las observaciones están equilibradas antes y después de la implantación del programa, en 2001.

Una vez seleccionados los indicadores se procedió a la aplicación del modelo utilizando la especificación general presentada en la ecuación [1] utilizándose las siguientes variables exógenas:

- $\quad G_{\text {: }}$ Toma el valor 1 para los municipios que tienen algún proyecto aprobado en el Plan Izartu y cero para el resto.(Ver gráfico 1)

- $\quad \mathbf{D}_{\mathbf{t}}$ : Toma el valor cero para los años anteriores al 2001 y 1 para el resto.

- $\quad \mathrm{T}_{\mathrm{j} \text { : }}$ Toma el valor 1 para los datos de los municipios con programas Izartu a partir del año 2001 y 0 en el resto.

En todos los casos el grupo de control está constituido por el resto de los 202 municipios del País Vasco que no se presentaron a esta primera convocatoria del programa Izartu.

Teniendo en cuenta la cantidad de información manejada (250 municipios por 8 años) se ha optado por estimar el modelo mediante datos de panel. A continuación, se exponen los resultados por las 5 categorías descritas 
anteriormente: infraestructuras (M1), actividad económica (M2), cohesión (M3), demografía (M4) y entidades locales (M5).

TABLA 4

RESULTADOS DEL MODELO DE DIFERENCIAS EN DIFERENCIAS DEL PLAN IZARTU (I)

\begin{tabular}{|c|c|c|c|c|c|}
\hline \multirow{2}{*}{ Modelo } & Variable & $\mathrm{a}$ & $\mathrm{b}$ & $\mathrm{g}$ & $\mathrm{d}$ \\
\hline \multirow{2}{*}{ M1 } & CPO & $3,44^{*}$ & $-2,39 *$ & 0,05 & 0,06 \\
\hline \multirow{2}{*}{ M2 } & EST & $80,2^{*}$ & $-6,1^{*}$ & 2,2 & 1,6 \\
\cline { 2 - 6 } & RPC & $8.953^{*}$ & $-519^{*}$ & $2.623^{*}$ & $-473^{*}$ \\
\hline \multirow{2}{*}{ M3 } & IRE & $112,3^{*}$ & $-13,9 *$ & $3,7^{*}$ & $-3,1^{*}$ \\
\cline { 2 - 6 } & TPA & $0,026^{*}$ & $0,017^{*}$ & $-0,004^{*}$ & $-0,005^{*}$ \\
\hline M4 & SMI & $1,34^{*}$ & $-2,1^{*}$ & $3,05^{*}$ & 0,84 \\
\hline \multirow{2}{*}{ M5 } & GTO & $1.008^{*}$ & $-352^{*}$ & $383^{*}$ & 27 \\
\cline { 2 - 6 } & GCAP & $441^{*}$ & $-284^{*}$ & $191^{*}$ & 30 \\
\hline
\end{tabular}

* Significativo al 95 \% de nivel de confianza.

TABLA 5

RESULTADOS DEL MODELO DE DIFERENCIAS EN DIFERENCIAS DEL PLAN IZARTU (II)

\begin{tabular}{|c|c|c|c|c|c|}
\hline \multicolumn{6}{|c|}{ Valores medios } \\
\hline \multirow[t]{2}{*}{ Modelo } & \multirow[t]{2}{*}{ Variable } & \multicolumn{2}{|c|}{$1997-2000$} & \multicolumn{2}{|c|}{ 2001-2004 } \\
\hline & & Resto & Izartu & Resto & Izartu \\
\hline M1 & $\mathrm{CPO}$ & 3,44 & 1,05 & 3,49 & 1,16 \\
\hline \multirow[t]{2}{*}{ M2 } & EST & 80,2 & 74,1 & 82,4 & 77,9 \\
\hline & $\mathrm{RPC}$ & 8.953 & 8.434 & 11.576 & 10.584 \\
\hline \multirow[t]{2}{*}{ M3 } & IRE & 112,3 & 98,4 & 116,0 & 99,0 \\
\hline & TPA & 2,6 & 4,3 & 2,2 & 3,4 \\
\hline M4 & SMI & 1,34 & $-0,76$ & 4,39 & 3,13 \\
\hline \multirow[t]{2}{*}{ M5 } & GTO & 1.008 & 656 & 1.391 & 1.066 \\
\hline & GCAP & 441 & 157 & 632 & 378 \\
\hline
\end{tabular}

Para la primera de las categorías, Infraestructuras (M1), los coeficientes coeficiente $\alpha$ y $\beta$ son significativos a un $95 \%$ de nivel de confianza. Considerando los problemas de significatividad agudizados por el mayor volumen de las muestras de datos, podríamos afirmar que el programa Izartu ha tenido un efecto positivo sobre las dotaciones de infraestructuras municipales, habiendo contribuido a aumentar ligeramente la dotación de los municipios incluidos en el programa, y que partían con una situación sensiblemente deficitaria con algo más de un centro por cada mil habitantes $(1,05)$ mientras que el resto de municipios contaban con 3,44.

En cualquier caso, y a pesar del efecto diferencial del programa, los niveles de dotación siguen siendo insuficientes, pues continúan situados en 1,16 centros por cada mil habitantes frente a los 3,49 que presentan, como media, el resto de municipios.

En la dimensión de Actividad Económica (M2) los resultados son dispares, ya que el programa Izartu refleja un efecto positivo sobre el número de establecimientos productivos, habiéndose incrementado de forma diferencial en 1,6 establecimientos por cada 1000 habitantes, mientras que en el caso de la renta bruta no se habrían registrado efectos positivos, e incluso los municipios con programas Izartu habrían aumentado su renta disponible en menor medida que el resto.

En la categoría de cohesión (M3) nos encontramos, nuevamente, con resultados dispares entre los dos indicadores utilizados. Por un lado, el índice de renta relativo presenta un cierto deterioro en el conjunto de municipios con programas Izartu; y, por otro, la tasa de paro refleja un efecto positivo y estadísticamente significativo. 
Así, los municipios incluidos en el programa partían con tasas de paro sensiblemente superiores al resto (4,3\% frente al 2,6\%), éstas se habrían visto reducidas en mayor medida en estos 48 municipios alcanzando un promedio del 3,4\% frente al 2,2\% del resto, es decir, se habría reducido la brecha en torno al medio punto. En el caso de los indicadores de renta, tanto el absoluto que analizamos en $\mathrm{M} 2$, como el relativo, sería interesante profundizar en los resultados obtenidos ya que son incongruentes ${ }^{8}$.

Los efectos demográficos (M4) resultan significativos, ya que, mientras en los periodos previos a la implantación del programa el conjunto de municipios Izartu presentaban saldos migratorios negativos (-0,76 frente a 1,34 del resto), tras la puesta en marcha de los programas ya presentan valores positivos habiéndose aproximado al comportamiento medio del resto de municipios (3,13 frente a 4,39).

Es decir, la implantación de programas Izartu han contribuido a fijar las poblaciones de los municipios participantes y a aprovechar de forma diferencial los flujos migratorios registrados en el conjunto del territorio durante los últimos años.

Finalmente, en la categoría de las entidades locales (M5), medida a través de los indicadores de gastos totales y gastos de capital per cápita, los efectos del programa Izartu parecen haber sido igualmente positivos, ya que se registra un aumento del nivel de gasto de forma diferencial en unos 30 euros por habitante y año.

Una vez más el nivel de partida de los indicadores analizados era sensiblemente inferior en el conjunto de municipios con programas Izartu, ya que presentaban un nivel de gasto total en torno al $65 \%$ del resto (656 frente a 1008), reduciéndose ese porcentaje hasta el 36\% (157 frente a 441) en el caso de los gastos de capital.

Tras la aplicación del programa esta brecha se ha reducido en el caso de los gastos totales que se sitúan al $77 \%$ de los presentados por el resto de municipios (1066 frente a 1391) y de forma mucho más significativa en el caso de los gastos de capital, donde el porcentaje se ha elevado hasta el 60\% (378 frente a 632).

A modo de resumen final podríamos afirmar que la implantación de los programas Izartu se han realizado en municipios que partían de una situación claramente deficitaria en todos los indicadores utilizados y, salvo en el caso de la renta, han contribuido a reducir la brecha que los separaba del resto de municipios, cumpliéndose así el objetivo general de aumentar la cohesión y reducir las diferencias entre el conjunto de municipios del País Vasco.

\section{1.- Aplicación a políticas regionales: Plan Euskadi de Fomento de la Sociedad de la Información.}

Tal como adelantábamos, la ilustración de la aplicación sobre políticas de ámbito regional se realizará mediante la aplicación al Plan Euskadi de Fomento de la Sociedad de la Información.

Teniendo en cuenta los objetivos establecidos y los programas de actuación diseñados, las variables de respuesta más adecuadas para realizar la valoración de este programa están relacionadas con los niveles de penetración y uso de las TIC en el conjunto de hogares y empresas vascas.

El Instituto Nacional de Estadística publica, desde el año 2002, los resultados por Comunidades Autónomas de la Encuesta sobre el uso de TIC y comercio electrónico en las empresas y de la Encuesta sobre Equipamiento y Uso de Tecnologías de la Información y Comunicación en los hogares, donde se recogen un conjunto de indicadores generales sobre los niveles de penetración y uso de las TIC en las diferentes regiones españolas.

Para el caso de empresas la encuesta actualmente recoge un total de 80 indicadores, mientras que en el caso de los hogares en la actualidad se dispone de más de 280 indicadores El gran número de indicadores disponibles complica la selección de los mismos, por lo que hemos optado por realizar una filtro que consideramos como representativo y que refleja los tres niveles básicos que podemos denominar como Equipamiento, Uso básico y Uso avanzado de las TIC.

8 Un primer acercamiento es recordar que mientras que el resto de indicadores se obtienen de magnitudes directas, en el caso de la renta se ha realizado un proceso de estimación a partir de indicadores indirectos y que pueden no reflejar adecuadamente la dinámica de la misma. Por otra parte, si analizamos el tipo de acciones incluidas en el programa Izartu podremos comprobar que prácticamente ninguna de ellas tiene como objetivo directo la mejora de la renta per cápita y, si bien es cierto que todas ellas tienden a mejorar el entorno donde se desarrolla la actividad, al actuar sobre los factores estructurales, estos efectos indirectos pueden tener un periodo de maduración más largo y que no ha sido aún recogido por los datos. Adicionalmente, lo que sí parece claro es que los municipios donde se han implementado estos programas partían de una situación significativamente más baja en términos de renta, con casi 14 puntos menos que el resto de municipios, lo que puede suponer un cierto freno al crecimiento en un modelo como el experimentado durante los últimos años por la economía española, y la vasca, en donde la demanda está teniendo un protagonismo especial. 
En la siguiente tabla se recogen los indicadores concretos de cada uno de los grupos:

TABLA 3

SELECCIÓN DE INDICADORES PARA VALORAR EL DESARROLLO DEL PLAN EUSKADI A NIVEL REGIONAL

\begin{tabular}{|c|c|c|}
\hline & Empresas & Hogares \\
\hline $\begin{array}{l}\text { E q u i p a - } \\
\text { miento }\end{array}$ & $\begin{array}{l}\text { Empresas con conexión a Internet } \\
(\%) .\end{array}$ & $\begin{array}{l}\text { Hogares que disponen de acceso a } \\
\text { Internet (\%). }\end{array}$ \\
\hline Uso Básico & $\begin{array}{l}\text { Personal que utiliza ordenadores } \\
\text { conectados a Internet al menos una vez } \\
\text { por semana (\%). }\end{array}$ & $\begin{array}{l}\text { Personas que han utilizado Internet } \\
\text { en los últimos } 3 \text { meses (\%). }\end{array}$ \\
\hline $\begin{array}{l}\text { Uso Avan- } \\
\text { zado }\end{array}$ & $\begin{array}{l}\text { Empresas que interactúan con las } \\
\text { Administraciones Públicas mediante } \\
\text { Internet (\%) y empresas con conexión a } \\
\text { Internet y sitio/página Web (\%). }\end{array}$ & $\begin{array}{l}\text { Personas que han comprado a tra- } \\
\text { vés de Internet en los últimos } 3 \text { meses } \\
\text { (\%). }\end{array}$ \\
\hline
\end{tabular}

Con el fin de realizar una primera valoración del impacto del Plan Euskadi de fomento de la Sociedad de la información se ha calculado un indicador sintético simple para la sociedad de la información (ISSI) obtenido por promedio directo entre los indicadores de empresa y los de hogares y una vez estandarizados cada uno de los indicadores individuales tomando como base de referencia el promedio nacional en el año 2002. Así, el horizonte temporal del modelo aplicado para la evaluación de este programa está definidlo entre los años 2002-2006.

Utilizando los datos de este ISSI para el conjunto de las 17 Comunidades Autónomas de España más las ciudades autónomas de Ceuta y Melilla durante los años 2002 a 2006, hemos realizado una valoración del Programa Euskadi de fomento de la Sociedad de la Información, utilizando las expresión [1] y asumiendo que los efectos del mismo se empezaron a registrar a partir del año 2004.

Así, en esta ocasión, la variable endógena, $\mathbf{Y}_{\mathrm{i} j \mathrm{t}, \mathrm{t}}$, es el Indicador Sintético de Sociedad de la Información registrado para las 18 regiones de España i, durante los años 2002,2003,2004,2005 y 2006, donde:

- $\quad G_{j}$ : Toma el valor 1 para las observaciones del País Vasco y cero en el resto.

- $\quad$ D: $_{\mathrm{t}}$ Toma el valor cero para los años 2002 y 2003 y 1 para el 2004,2005 y 2006.

- $\quad \mathbf{T}_{\mathrm{jit}}$ : Toma el valor 1 para los datos del País Vasco en 2004,2005 y 2006 y 0 en el resto.

Una vez estimado el modelo por el procedimiento de MCO los resultados obtenidos fueron los siguientes:

TABLA 6

VALORACIÓN GENERAL DEL PROGRAMA EUSKADI A NIVEL REGIONAL.

\begin{tabular}{|c|c|c|}
\hline Coeficiente & Valor & Significatividad \\
\hline$\alpha$ & 117,7 & $12,4^{*}$ \\
\hline $\mathrm{b}$ & 25,3 & 0,6 \\
\hline$\gamma$ & 81,6 & $6,7^{*}$ \\
\hline$\delta$ & 35,3 & 0,7 \\
\hline
\end{tabular}

* Significativo al $95 \%$ s de nivel de confianza.

Antes de la implantación del programa Euskadi de fomento de la S.I., (entre los años 2002 y 2003 ) el conjunto de Comunidades Autónomas presentaban un desarrollo medio de un 18\% por encima del año base ( $a=117,7$ sobre un índice Media 2002 =100) mientras que en ese mismo periodo el País Vasco ya partía con un cierto diferencial positivo alcanzado un índice medio ligeramente superior a $143^{9}$.

Después de la implantación del programa, (entre los años 2004 a 2006) el promedio de las regiones alcanzaron un desarrollo de la S.I. equivalente a casi el doble del registrado en el 2002, con un índice medio de $199,7^{10}$.

\footnotetext{
$9 \alpha+b=117,7+25,3=143,1$.
}

$10 \alpha+\gamma=117,7+81,6=199,4$. 
En ese mismo periodo, el País Vasco presentaba un índice en torno a $260^{11}$. De esta forma podríamos admitir que la puesta en marcha del programa Euskadi de fomento de la S.I. habría contribuido a aumentar el índice medio de desarrollo de la S.I. en unos 35 puntos.

En cualquier caso, y aún teniendo en cuenta todos los matices comentados en la parte metodológica de este artículo sobre la significatividad estadística de los coeficientes de los modelos DiD, debemos poner de manifiesto que, ni el coeficiente b que recoge la diferencia de partida, ni el d que refleja los efectos del tratamiento, resultan estadísticamente significativos por lo que los resultados obtenidos deberían ser interpretados de forma cautelosa.

En una segunda etapa se diferenciaron los indicadores específicos de empresas y hogares obteniéndose los resultados que analizamos a continuación.

TABLA 7

VALORACIÓN DIFERENCIADA DEL PROGRAMA EUSKADI

\begin{tabular}{|l|c|c|c|c|}
\hline \multicolumn{5}{|c|}{ Coeficientes estimados } \\
\hline & $\alpha$ & $\beta$ & $\gamma$ & $\delta$ \\
\hline Empresas & $101,5^{*}$ & $19,6^{*}$ & $15,1^{*}$ & $-6,7$ \\
\hline Hogares & $134,2^{*}$ & 30,8 & $115,6 *$ & 35,2 \\
\hline \multicolumn{4}{|c|}{ Valores medios } \\
\hline \multicolumn{2}{|c|}{$2002-2003$} & Resto CC.AA. & C.A.P.V. \\
\hline & Resto CC.AA. & C.A.P.V. & 116,6 & 129,6 \\
\hline Empresas & 101,5 & 121,2 & 249,8 & 315,8 \\
\hline Hogares & 134,2 & 165,0 &
\end{tabular}

* Significativo al $95 \%$ s de nivel de confianza.

El Plan Euskadi de fomento de la S.I. tiene mayor impacto en los hogares que en las empresas, ya que mientras en las familias habría tenido un efecto neto de avance de más de 35 puntos, en el caso de las empresas no habría conseguido ni siquiera mantener el mismo ritmo de expansión que el resto de las Comunidades Autónomas.

En cualquier caso, el País Vasco sigue presentando un importante diferencial del desarrollo de la S.I., tanto en el ámbito empresarial, con más de trece puntos por encima de la media del resto de comunidades, como en los hogares, donde el diferencial se amplía a 66 puntos.

En términos de la efectividad del programa analizado, sobre todo en el ámbito empresarial en donde no parece presentar buenos resultados, deben tenerse en cuenta una serie de factores que estarían afectando a los resultados obtenidos. En primer lugar el mayor nivel de desarrollo de partida que presentaba el País Vasco hace que los avances relativos sean progresivamente más costosos al evolucionar de acuerdo con procesos de difusión acotados.

Por otro lado debemos tener en cuenta la existencia de otros programas similares en el resto de comunidades autónomas y que podrían estar acelerando la dinámica relativa de los indicadores analizados.

Así, en Pulido et. al (2001) podemos encontrar programas específicos de fomento de la sociedad de la información en Cataluña (2000-2001), Extremadura, Galicia (2001-2004), La Rioja (2000-2003) y Navarra(2001-2003); mientras que en otras tantas comunidades existían iniciativas o programas que incidían parcialmente en algunos aspectos concretos de estas nuevas tecnologías (Andalucía, Canarias, Comunidad Valenciana y Castilla y León).

Tratando de obtener un mayor detalle en el proceso de evaluación se han realizado modelos de evaluación para cada indicador individual, tanto para las empresas como para los hogares, encontrando los siguientes resultados.

$11 a+b+g+d=117,7+25,3+81,6+35,3=260,0$ 
TABLA 8

VALORACIÓN SOBRE LOS INDICADORES DE EMPRESAS Y HOGARES (I)

\begin{tabular}{|l|l|l|l|l|l|l|l|l|}
\hline \multicolumn{1}{|c|}{ Coeficientes estimados } \\
\hline \multirow{2}{*}{ Indicadores } & \multicolumn{9}{|c|}{ Empresas } & \multicolumn{1}{c|}{ Hogares } \\
\cline { 2 - 11 } & \multicolumn{1}{|c|}{$\alpha$} & \multicolumn{1}{c|}{$\beta$} & $\gamma$ & $\delta$ & $\alpha$ & $\beta$ & $\gamma$ & $\delta$ \\
\hline Equipamiento & $83,7^{*}$ & 5,7 & $8,2^{*}$ & $-2,4$ & $20,2^{*}$ & 6,9 & $9,6^{*}$ & 3,4 \\
\hline Uso básico & $23,3^{*}$ & $8,3^{*}$ & $6,0^{*}$ & $-1,4$ & $25,2^{*}$ & 3,4 & $13,1^{*}$ & 1,7 \\
\hline Uso Avanzado & $47,2^{*}$ & 7,7 & $7,2^{*}$ & $-2,0$ & 2,9 & 0,7 & 2,6 & 2,3 \\
\hline
\end{tabular}

* Significativo al $95 \%$ s de nivel de confianza.

TABLA 9

VALORACIÓN SOBRE LOS INDICADORES DE EMPRESAS Y HOGARES (II)

\begin{tabular}{|c|c|c|c|c|c|c|c|c|}
\hline \multicolumn{9}{|c|}{ Valores Medios } \\
\hline \multirow{3}{*}{ Indicadores } & \multicolumn{4}{|c|}{ Empresas } & \multicolumn{4}{|c|}{ Hogares } \\
\hline & \multicolumn{2}{|c|}{$2002-2003$} & \multicolumn{2}{|c|}{ 2004-2006 } & \multicolumn{2}{|c|}{$2002-2003$} & \multicolumn{2}{|c|}{$2004-2006$} \\
\hline & $\begin{array}{l}\mathrm{R} \text { e s t o } \\
\text { regiones }\end{array}$ & $\begin{array}{l}\text { P a ís } \\
\text { Vasco }\end{array}$ & $\begin{array}{l}\mathrm{R} \text { e s t o } \\
\text { regiones }\end{array}$ & $\begin{array}{l}\text { P a í s } \\
\text { Vasco }\end{array}$ & $\begin{array}{l}\mathrm{R} \text { e s t o } \\
\text { regiones }\end{array}$ & $\begin{array}{l}\text { P a ís } \\
\text { Vasco }\end{array}$ & $\begin{array}{l}\mathrm{R} \text { e s t o } \\
\text { regiones }\end{array}$ & $\begin{array}{l}\text { P a í s } \\
\text { Vasco }\end{array}$ \\
\hline Equipamiento & 83,7 & 89,4 & 91,8 & 95,2 & 20,2 & 27,0 & 29,8 & 40,1 \\
\hline Uso básico & 23,3 & 31,6 & 29,3 & 36,2 & 25,2 & 28,6 & 38,4 & 43,4 \\
\hline Uso Avanzado & 47,2 & 55,0 & 54,4 & 60,2 & 2,9 & 3,6 & 5,6 & 8,6 \\
\hline
\end{tabular}

En general, ninguno de los indicadores seleccionados para el conjunto de empresas estaría reflejando efectos significativos de la implantación del programa. Sin embargo, en todos los casos se mantiene el patrón general observado para el indicador sintético de empresas en el sentido de presentar un avance diferencial en País Vasco frente a la media del resto de Comunidades Autónomas.

De hecho, tanto en los indicadores de uso básico, como en los de uso avanzado, la posición media del resto de comunidades en el periodo 2004-2006 es inferior a la que presentaba el País Vasco en el periodo precedente 2002-2003.

En cualquier caso, durante el periodo analizado se han registrado avances en todos los indicadores en el País Vasco, habiéndose aumentado en 6 puntos el porcentaje de empresas conectadas a Internet, en algo menos de 5 puntos el porcentaje de empleados que utilizan ordenadores conectados a Internet al menos un vez por semana, y en otros 5 puntos el porcentaje de empresas que disponen de página web activa.

En cuanto a los hogares, los resultados son parecidos al análisis del indicador sintético y es que el programa Euskadi de fomento de la S.I., sí habría tenido un efecto diferencial sobre los hogares vascos, aunque siguen persistiendo los problemas de significatividad estadística de los coeficientes estimados.

Por otra parte, el porcentaje de hogares que disponen de acceso a Internet se habría incrementado de forma significativa en 3,4 puntos, hasta situarse por encima del 40\% del total frente al 29,8 de media en el resto de Comunidades Autónomas.

En cuanto al porcentaje de personas que han utilizado Internet en los últimos tres meses, éste se habría visto incrementado en 1,7 puntos diferenciales por efecto del programa analizado, situándose en el 43,4\% frente al 38,4\% del resto de regiones.

Finalmente, el porcentaje de población que ha realizado compras a través de la red en los últimos tres meses, se habría visto dinamizado en 2,3 puntos, situándose en niveles del 8,6\% frente al 5,6\% del resto. 


\section{5.- VALORACIÓN DE RESULTADOS Y PROPUESTAS ALTERNATIVAS}

Una vez revisadas las diferentes tipologías de políticas públicas desarrolladas por las administraciones territoriales, ejemplificadas a través de la Comunidad Autónoma del País Vasco, hemos podido constar que la propuesta metodológica de evaluación basada en los modelos de diferencias en diferencias tiene una elevada potencialidad de aplicación, aun cuando no se disponga de la información microeconómica con la que habitualmente se asocia este tipo de técnicas.

En efecto, el progresivo desarrollo de las estadísticas territoriales ofrece, en la actualidad, una batería suficiente de información que permite la realización de aplicaciones de evaluación de políticas públicas en el ámbito regional y local, tal como se ha ilustrado con los dos ejemplos realizados.

De esta forma, la primera conclusión relevante del trabajo realizado iría, precisamente, en la línea potenciar el desarrollo y recomendar el uso de este tipo de metodologías que permiten, de una forma relativamente sencilla, cuantificar y visualizar los efectos de estas políticas.

Ahora bien, una vez establecida la potencialidad de esta metodología de los modelos de diferencias en diferencias, es necesario, también, dejar constancia de las limitaciones que presentan cuando son aplicadas a datos agregados como los que se ilustran en el presente artículo.

Tal como se ha recogido en los párrafos precedentes, la primera de estas limitaciones estaría vinculada con la selección del grupo de control, ya que, como hemos visto, no siempre es posible seleccionar un conjunto alternativo de observaciones muestrales que sigan, a priori, una evolución similar a las del grupo de tratamiento y donde no se haya aplicado la política concreta a analizar, bien sea porque dichas políticas hayan sido aplicadas de forma extensiva a toda la muestra (p.e. políticas locales aplicadas a la totalidad de municipios), o bien, porque el grupo de control pueda estar afectado por otras actuaciones no contempladas en el modelo (p.e. la aplicación de programas similares en otras regiones).

Adicionalmente habría que añadir la posible existencia de desfases temporales entre la aplicación de la política analizada y la materialización de los efectos buscados y que, en algunas ocasiones, puede superar los horizontes de disponibilidad temporal de la información agregada. (p.e. políticas educativas de largos procesos de maduración).

Junto a estas limitaciones detectadas en las aplicaciones sobre datos agregados habría que incluir los posibles efectos del incumplimiento de las condiciones de identificación comunes a todas las aplicaciones de los modelos de diferencias en diferencias.

Estas condiciones de identificación se concretan, como comentamos en el apartado metodológico, en la estabilidad de composición y la tendencia paralela.

En el primer caso, la condición de estabilidad en la composición, el modelo asume que ambos grupos, el de tratamiento y el de control, atraen al mismo número de individuos con independencia de que se produzca, o no, el tratamiento; concretándose en una expresión del tipo:

$$
E\left(Y_{j, 0} \mid D_{t}=0, G_{j}=j\right)=E\left(Y_{j, 0} \mid D_{t}=1, G_{j}=j\right) \forall j=0,1[7]
$$

En términos prácticos, esta condición supone que el hecho de aplicar una determinada política no aumenta (o disminuye) el grupo de individuos a la que se aplica, por lo que en el caso de las aplicaciones sobre datos agregados no tiene, en principio, una incidencia significativa ya que supondría, por ejemplo, un cambio de adscripción de un municipio de una comunidad a otra por el hecho de que existiera una determinada política local.

Por el contrario, la condición de tendencia paralela, que se concreta mediante una expresión como la que recogemos a continuación, sí que puede tener una influencia mayor en las aplicaciones sobre datos agregados.

$$
E\left(Y_{i, G=1, D=1 \sin \text { tratamiento }}-Y_{i, G=1, D=0} \mid G_{j}=1\right)=E\left(Y_{i, G=0, D=1}-Y_{i, G=0, D=0} \mid G_{j}=0\right)[8]
$$

En efecto, esta condición supone que ambos grupos, el de tratamiento y el de control, seguirían evolución temporal similar en los valores de la variable objetivo si no se produjera el tratamiento.

Ahora bien, los problemas detectados en la aplicación, tanto al programa Izartu, como al Plan Euskadi de fomento de la S.I. nos han llevado a identificar algunas situaciones en las que el modelo puede presentar resultados 
sesgados precisamente en aquellos casos en los que la evolución de los indicadores seleccionados no es estrictamente lineal y los niveles de partida en los grupos de tratamiento y de control son sensiblemente diferentes.

Así, cuando aplicamos el modelo en su planteamiento más sencillo, estamos asumiendo que el avance que seguirían los indicadores en el grupo de tratamiento en ausencia del mismo (sin la aplicación de la política analizada) sería de la misma magnitud que en el grupo de control. (Evaluación contrafactual), tal como se ilustra en la tabla que presentamos a continuación.

TABLA 10.

EJEMPLO DE EFECTOS SOBRE UNA VARIABLE DE RENTA PER CÁPITA

\begin{tabular}{|l|r|r|r|r|r|r|}
\hline & \multicolumn{1}{|c|}{ Antes } & \multicolumn{1}{c|}{ Después } & Variación & Efecto & \% Cto. & Diferencia \\
\hline Grupo de control & 12.000 & 16.000 & 4.000 & & $33 \%$ & \\
\hline Grupo de tratamiento & 8.000 & 11.200 & 3.200 & -800 & $40 \%$ & $7 \%$ \\
\hline Contrafactual (\% cto.) & 8.000 & 10.667 & 2.667 & 533 & & \\
\hline
\end{tabular}

De acuerdo con los datos presentados en la tabla anterior, el conjunto de individuos incluidos en el grupo de tratamiento habrían incrementado su renta disponible en $3.200 €$ tras la aplicación del tratamiento, mientras que los del grupo de control lo habrían hecho en $4.000 €$ de forma tal que el efecto neto habría sido, aparentemente negativo $(-800 €)$.

Ahora bien, si realizáramos una evaluación contrafactual considerando que el grupo de tratamiento habría tenido un crecimiento de la renta disponible similar al del grupo de control (33\%), el nivel de renta esperado tras la aplicación del tratamiento habría sido de $10.667 €$, de lo que se deduciría que el efecto neto habría sido positivo $(3.200-2.667=533)$.

Este tipo de discrepancias se generan cuando las variables objetivo no presentan tendencias lineales y los niveles absolutos de partida de los grupos de tratamiento y de control son muy diferentes, es decir, que no es lo mismo un crecimiento del $5 \%$ sobre una magnitud de 100 , es decir, 5 unidades, que sobre una magnitud de 50 ( 2,5 unidades), tal como se ilustra en el gráfico que presentamos a continuación, donde se recoge la evolución del grupo de control (el de nivel más elevado), la del grupo de tratamiento (el de nivel más bajo) asumiendo el cumplimiento estricto de la condición de tendencia paralela, es decir, con aumentos absolutos similares a los del grupo de control, y la denotada como contrafactual que resultaría de aplicar al grupo de tratamiento la misma tasa de crecimiento que el grupo de control.

Por diferencia entre la evolución asumida por la condición de tendencia paralela en sentido estricto y la contrafactual, que asume la igualdad en las tasas de crecimiento, podemos estimar el sesgo que se generaría por la aplicación del modelo de diferencias en diferencias en su formulación general. 
FIGURA 2

ILUSTRACIÓN DEL SESGO INDUCIDO POR LA CONDICIÓN DE TENDENCIA PARALELA EN VARIABLES CON TENDENCIA NO LINEAL

(ASUMIENDO TASAS DE CRECIMIENTO CONSTANTES DEL 5\%)
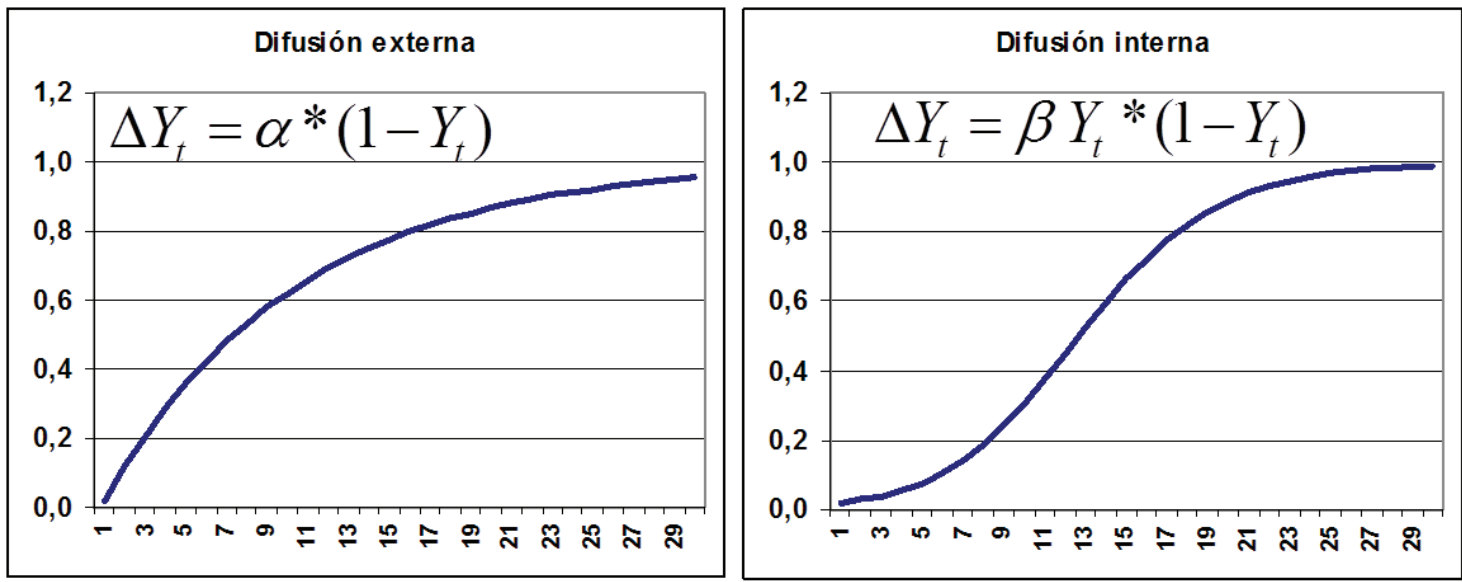

Como puede constatarse en el gráfico anterior, ante la existencia de tendencias no lineales con tasa de crecimiento estacionarias, el sesgo en la evaluación será tanto mayor cuanto mayor sea la diferencia de partida en los niveles de la variable objetivo del grupo de tratamiento y el grupo de control y más se alargue el periodo de evaluación.

En la misma línea, y tal como hemos podido comprobar en la aplicación realizada sobre la política de fomento de la sociedad de la información, algunos de los indicadores utilizados presentan procesos acotados de evolución, tales como el porcentaje de usuarios de nuevas tecnologías, o el número de líneas digitales por cada 1000 habitantes, y que responden, en general, a los denominados procesos de difusión cuya expresión matemática y evolución temporal presentamos a continuación.

FIGURA 3

\section{ESPECIFICACIÓN Y EVOLUCIÓN DE LOS PROCESOS DE DIFUSIÓN ACOTADOS}
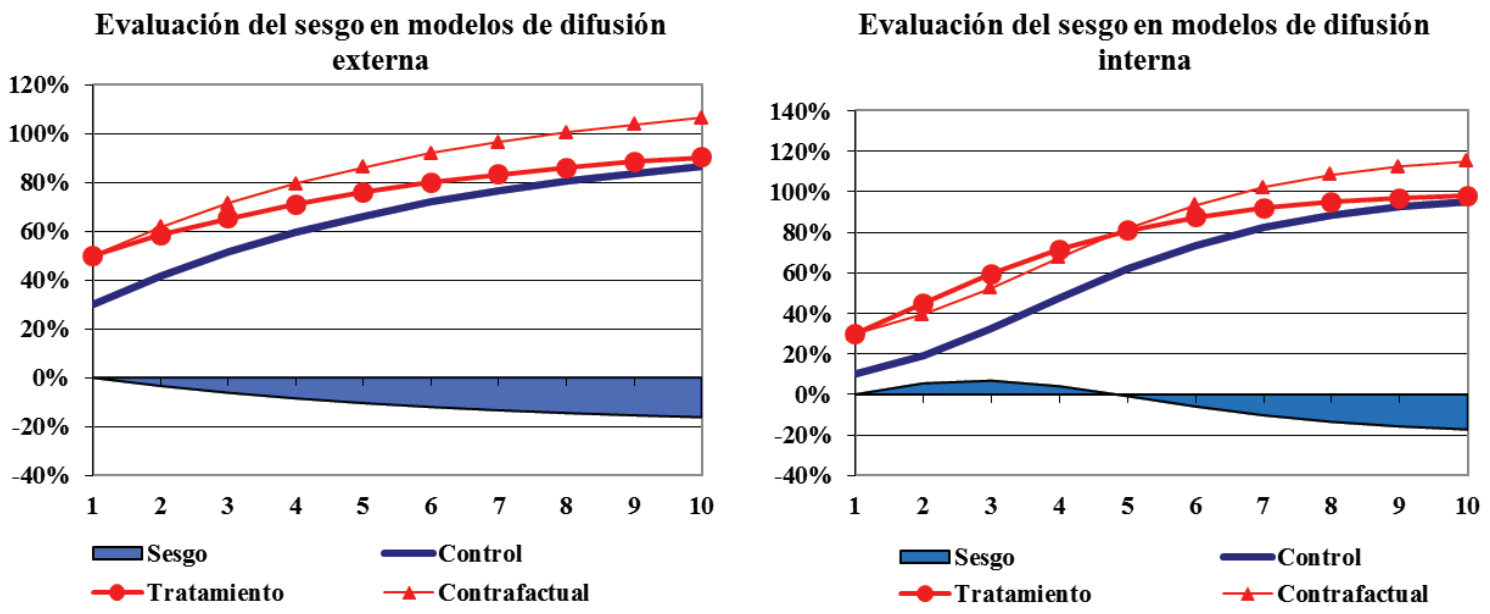

En estos casos, la aplicación de la condición de tendencia paralela generaría también sesgos en los resultados de la evaluación que dependerían, tanto de la posición inicial de los grupos de tratamiento y control, como del tipo de procesos de difusión seguidos, tal como se ilustra a continuación. 
FIGURA 4

ILUSTRACIÓN DEL SESGO INDUCIDO POR LA CONDICIÓN DE TENDENCIA PARALELA

EN VARIABLES CON PROCESOS DE DIFUSIÓN ACOTADOS

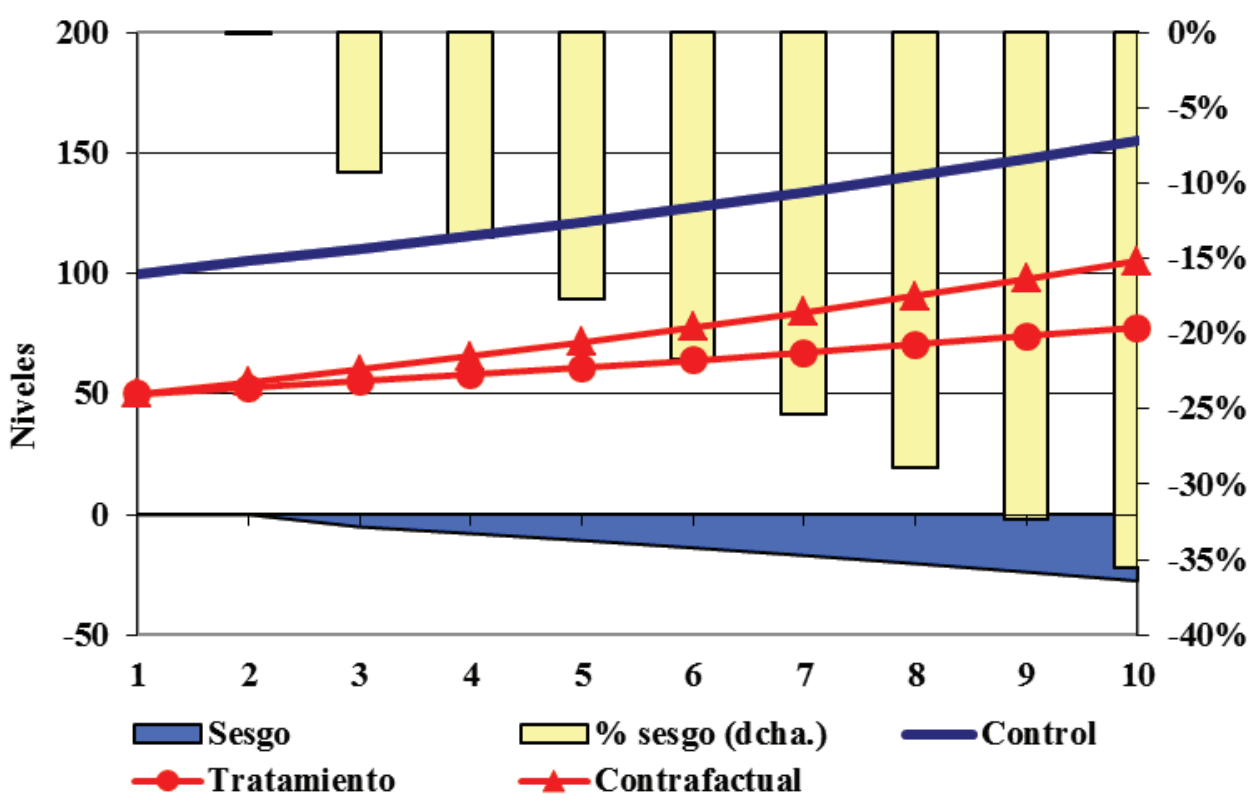

Tal como puede comprobarse en la ilustración precedente, en este tipo de procesos la aplicación estricta de la condición de tendencia paralela penalizaría, en todos los casos, a los grupos más avanzados en los modelos de difusión externa, mientras que en los modelos de influencia interna, se generaría un sesgo positivo para los grupos de niveles superiores durante los primeros periodos que se tornaría negativo y creciente una vez superado el punto de inflexión de la curva.

Frente a estas circunstancias, una posible solución consistiría en realizar la evaluación contrafactual utilizando, no la expresión lineal derivada del modelo general, sino una transformación de la misma de acuerdo con la evolución estimada del indicador.

Así, por ejemplo, si admitimos que las tendencias no son lineales sino que ambos grupos (tratamiento y control) deberían presentar una dinámica similar en ausencia del tratamiento, es decir, tasas de crecimiento similares, tendríamos que admitir que el valor esperado en el grupo de tratamiento vendría dado por:

$$
\hat{\delta}=\left(\bar{Y}_{G=1, D=1}-\bar{Y}_{G=1, D=1 \text { sin tratamiento }}\right)[9]
$$

siendo:

$$
\bar{Y}_{G=1, D=1 \sin \text { tratamiento }}=\bar{Y}_{G=1, D=0} *\left(\frac{\bar{Y}_{G=0, D=1}}{\bar{Y}_{G=0, D=0}}\right)[10]
$$

En lugar de la especificación general de cumplimiento estricto de la condición de tendencia paralela.

$$
\bar{Y}_{G=1, D=1 \sin \text { tratamiento }}=\bar{Y}_{G=1, D=0}+\left(\bar{Y}_{G=0, D=1}-\bar{Y}_{G=0, D=0}\right)[11]
$$

En el caso de indicadores acotados que siguen procesos de difusión la corrección sería algo más complicada.

Así, para los modelos de influencia externa se puede realizar una estimación de coeficiente de difusión a a partir de los datos del grupo de control mediante una expresión del tipo: 


$$
\alpha=\frac{\bar{Y}_{G=0, D=1}-\bar{Y}_{G=0, D=0}}{1-\bar{Y}_{G=0, D=1}}[12]
$$

Y a continuación calcular el valor contrafactual como:

$$
\bar{Y}_{G=1, D=1 \sin \text { tratamiento }}=\frac{\left(\bar{Y}_{G=1, D=0}+\alpha\right)}{(1-\alpha)}[13]
$$

Obteniéndose el efecto del tratamiento, o política aplicada, por diferencia entre el valor observado y el contrafactual:

$$
\hat{\delta}=\left(\bar{Y}_{G=1, D=1}-\bar{Y}_{G=1, D=1 \text { sin tratamiento }}\right)[14]
$$

Para los modelos de influencia interna la derivación del coeficiente $\beta$ asociado al proceso de difusión se obtendría como:

$$
\beta=\frac{\bar{Y}_{G=0, D=1}-\bar{Y}_{G=0, D=0}}{\bar{Y}_{G=0, D=1} *\left(1-\bar{Y}_{G=0, D=1}\right)}[15]
$$

Mientras que el valor contrafactual se obtendría de la solución de una ecuación de segundo grado que podría arrojar valores no reales.

$$
\begin{aligned}
& \beta *\left(\bar{Y}_{G=1, D=1}\right)^{2}+(1-\beta) * \bar{Y}_{G=1, D=1}-\bar{Y}_{G=1, D=0}=0[16] \\
& \bar{Y}_{G=1, D=1 \text { sin tratamiento }}=\frac{-(1-\beta) \pm \sqrt{(1-\beta)^{2}+4 * \beta * \bar{Y}_{G=1, D=0}}}{\left(2^{*} \beta\right)}[17]
\end{aligned}
$$

Dada la mayor dificultad de cómputo de esta expresión y el hecho de que pueda ofrecer resultados el campo no real (raíces complejas), a efectos prácticos y asumiendo que los procesos responden a una especificación logística normal con el punto de inflexión en la mitad del recorrido total, podría utilizarse una corrección alternativa como la que proponemos a continuación para el cálculo del valor contrafactual:

$$
\bar{Y}_{G=1, D=1 \text { sin tratamiento }}=\bar{Y}_{G=1, D=0} *\left[1+\left(\frac{\bar{Y}_{G=0, D=1}}{\bar{Y}_{G=0, D=0}}-1\right) * 2 *\left(1-\bar{Y}_{G=1, D=0}\right)\right]
$$

A modo de resumen final, debemos reiterar que las aplicaciones realizadas del modelo de diferencias en diferencias utilizando datos agregados para la evaluación de políticas públicas han arrojado resultados suficientemente interesantes y consistentes como para incorporar este tipo de metodologías en la batería general de técnicas de evaluación de estas políticas, si bien, es necesario tener en cuenta las limitaciones recogidas a lo largo del presente artículo y, de manera especial, los problemas derivados de la aplicación estricta de la condición de tendencia paralela cuando se utilizan este tipo de datos agregados, aconsejándose la aplicación de las correcciones del sesgo propuestas en el último apartado cuando las variables objetivo a analizar no presentan tendencias lineales. 


\section{BIBLIOGRAFÍA}

Acs, G., \& Nelson, S. (2004). Changes in living arrangements during the late 1990s: do welfare policies matter?. Journal of Policy Analysis and Management, 23(2), 273-290.

Banzhaf, H.S. y Walsh, R. (2006): “Do People Vote with Their Feet? An Empirical Test of Environmental Gentrification", Resources For the Future, Discussion Papers. http://www.rff.org/documents/RFF-DP-06-10.pdf

Bertrand, M., Duflo, E., \& Mullainathan, S. (2004). How much should we trust differences-in-differences estimates?. The Quarterly Journal of Economics, 119(1), 249-275.

Blundell, R., Dias, M. C., Meghir, C., \& Reenen, J. (2004). Evaluating the employment impact of a mandatory job search program. Journal of the European Economic Association, 2(4), 569-606.

Blundell, R., Duncan, A., \& Meghir, C. (1998). Estimating labor supply responses using tax reforms. Econometrica, 66(4), 827-861.

Card, D. (1989). The impact of the Mariel boatlift on the Miami labor market (No. w3069). National Bureau of Economic Research.

Card, D., \& Krueger, A. B. (2000). Minimum wages and employment: a case study of the fast-food industry in New Jersey and Pennsylvania: reply. American Economic Review, 90(5), 1397-1420.

Domino, M. E., Norton, E. C., Morrissey, J. P., \& Thakur, N. (2004). Cost shifting to jails after a change to managed mental health care. Health Services Research, 39(5), 1379-1402.

Douglas, S. (2006). Measuring Gains from Regional Dispatch: Coal-Fired Power Plant Utilization and Market Reforms. Energy Journal, 27(1), 119-138.

Duflo, E. (2000). Schooling and labor market consequences of school construction in Indonesia: Evidence from an unusual policy experiment (No. w7860). National Bureau of Economic Research.

Eissa, N., \& Liebman, J. B. (1996). Labor supply response to the earned income tax credit. The Quarterly Journal of Economics, 111(2), 605-637.

Feder, G., Murgai, R., \& Quizon, J. B. (2004). Sending farmers back to school: The impact of farmer field schools in Indonesia. Applied Economic Perspectives and Policy, 26(1), 45-62.

García Gómez, P. (2005). Evaluación de un programa de atención dental público: PADI en el País Vasco. Ekonomiaz, 60(2), 62-89.

Girma, S., \& Görg, H. (2007). Evaluating the foreign ownership wage premium using a difference-in-differences matching approach. Journal of International Economics, 72(1), 97-112.

Gorg, H., \& Strobl, E. (2005). Do government subsidies stimulate training expenditure? Microeconometric evidence from plant level data. Discusion Paper Series $n^{\circ} 1606$. Institute for the Study of Labor.

Gruber, J., \& Madrian, B. C. (1994). Health insurance and job mobility: The effects of public policy on job-lock. Industrial and Labor Relations Review, 48(1), 86-102.

Hamermesh, D. S., \& Scoones, W. D. (1999). Policy equilibria in a federal system: the effects of higher tax ceilings for unemployment insurance. Journal of Public Economics, 74(2), 191-213.

Hansson, A. (2008). The wealth tax and entrepreneurial activity. Journal of Entrepreneurship, 17(2), $139-156$.

Heckman, J. J., LaLonde, R. J., \& Smith, J. A. (1999). The economics and econometrics of active labor market programs. Handbook of labor economics, 3(A), 1865-2097.

Hoynes, H. W., Gelbach, J. B., \& Bitter, M. P. (2005). Welfare Reform and Health. Journal of human resources, 40(2), 309-334.

Konetzka, R. T., Yi, D., Norton, E. C., \& Kilpatrick, K. E. (2004). Effects of Medicare payment changes on nursing home staffing and deficiencies. Health services research, 39(3), 463-488.

Liu, Z., Dow, W. H., \& Norton, E. C. (2004). Effect of drive-through delivery laws on postpartum length of stay and hospital charges. Journal of Health Economics, 23(1), 129-155.

Neumark, D., \& Wascher, W. (2003). Minimum wages and skill acquisition: Another look at schooling effects. Economics of Education Review, 22(1), 1-10. 
Planas Miret, I. (2005). Principales mecanismos de evaluación económica de políticas públicas. Ekonomiaz, 60(1), 98-121.

Puhani, P. A. (2012). The treatment effect, the cross difference, and the interaction term in nonlinear "difference-in-differences" models. Economics Letters, 115(1), 85-87.

Pulido, A., López, A.M., Sánchez, J.C. y Lázarich, R. (2001): "Actuaciones regionales para la implantación de empresas", Documentos N-economía. Ceprede. Madrid.

Rubb, S. (2003). Social Security's Earnings Test Penalty and the Employment Rates of Elderly Men Aged 65 to 69. Eastern Economic Journal, 29(3), 415-431.

Shen, Y. C., \& Zuckerman, S. (2005). The effect of Medicaid payment generosity on access and use among beneficiaries. Health Services Research, 40(3), 723-744.

Shapiro, J., \& Skoufias, E. (2006). Evaluating the impact of Mexico's Quality Schools Program: The pitfalls of using nonexperimental data. World Bank Policy Research Working Paper 4036, October 2006

Smith, J., \& Todd, P. (2005). Does matching overcome LaLonde's critique of nonexperimental estimators?. Journal of econometrics, 125(1), 305-353.

Song, J. G. (2003). Evaluating the initial impact of eliminating the retirement earnings test. Social Security Bulletin, 65(1), 1-15.

Ten Cate, A. (2003). The Impact of Provincial Maternity and Parental Leave Policies on Employment Rates of Women with Young Children in Canada (No. 2003-03). 


\section{ANEXOS}

TABLA A1

POLÍTICAS LOCALES DE ACTUACIÓN DIRECTA

\begin{tabular}{|c|c|c|}
\hline Política & $\begin{array}{l}\text { Indicadores de } \\
\text { Intensidad / Uso }\end{array}$ & $\begin{array}{l}\text { Variables de res- } \\
\text { puesta }\end{array}$ \\
\hline \multicolumn{3}{|l|}{$\begin{array}{l}\text { Identificación de oportunidades de colabo- } \\
\text { ración interempresarial entre las empresas de Gi- } \\
\text { pukzkoa y del País Vasco del Norte }\end{array}$} \\
\hline \multicolumn{3}{|l|}{ Proceso de reflexión estratégica G2020 } \\
\hline \multicolumn{3}{|l|}{$\begin{array}{l}\text { II Plan de igualdad de oportunidades de Muje- } \\
\text { res y Hombres }\end{array}$} \\
\hline \multicolumn{3}{|l|}{ Plan de Acción de AL de Durango } \\
\hline \multicolumn{3}{|l|}{$\begin{array}{l}\text { Plan Director Participación Ciudadana Donos- } \\
\text { tia-San Sebastián }\end{array}$} \\
\hline \multicolumn{3}{|l|}{ Plan Estratégico Vitoria 2010} \\
\hline $\begin{array}{l}\text { Plan de Accesibilidad para los edificios de la Di- } \\
\text { putación Foral de Gipuzkoa }\end{array}$ & $\begin{array}{l}\mathrm{N}^{\circ} \text { de edificios acce- } \\
\text { sibles }\end{array}$ & \\
\hline Plan Territorial de Carreteras de Bizkaia & Km de carreteras & \\
\hline Plan Municipal de la Bicicleta en Donosita & Km vías, Uso & \\
\hline Plan de Movilidad Sostenible de Debagoiena & das $N^{\circ}$ acciones realiza- & \\
\hline Política Hidráulica de Bizkaia & das $\mathrm{N}^{\circ}$ acciones realiza- & \\
\hline Plan de Caminos Rurales 2007 & $\mathrm{Km}, \mathrm{N}^{\circ}$ municipios & \\
\hline Arasur & $\begin{array}{l}\text { Mercancías transpor- } \\
\text { tadas }\end{array}$ & \\
\hline Izartu II Donostia-San Sebastian & $\mathrm{N}^{\circ}$ acciones & $\begin{array}{l}\text { Percepción am- } \\
\text { biental }\end{array}$ \\
\hline $\begin{array}{l}\text { Planteamiento urbanístico y desarrollo soste- } \\
\text { nible }\end{array}$ & das $\mathrm{N}^{\circ}$ acciones realiza- & $\begin{array}{l}\text { Percepción am- } \\
\text { biental }\end{array}$ \\
\hline Bilbao Ría 2.000 & das $\mathrm{N}^{\circ}$ acciones realiza- & $\begin{array}{l}\text { Percepción am- } \\
\text { biental }\end{array}$ \\
\hline $\begin{array}{l}\text { II Plan Integral de Gestión de Residuos de Bi- } \\
\text { zkaia }\end{array}$ & das $\mathrm{N}^{\circ}$ acciones realiza- & $\begin{array}{l}\% \text { residuos recicla- } \\
\text { dos }\end{array}$ \\
\hline Plan de Gestión de calidad del aire & das $\mathrm{N}^{\circ}$ acciones realiza- & Emisiones $\mathrm{CO}_{2}$ \\
\hline Plan de Gestión de calidad del aire & das $\mathrm{N}^{\circ}$ acciones realiza- & Emisiones $\mathrm{CO}_{2}$ \\
\hline Zaintek & $\mathrm{N}^{\circ}$ usuarios & Competitividad \\
\hline
\end{tabular}

Fuente: Elaboración propia en base al SVDR.

Como puede comprobarse en la tabla anterior, las necesidades de información para realizar una evaluación de los efectos inducidos de estas políticas estarían vinculadas, fundamentalmente, con la disponibilidad de indicadores de tipo medioambiental a nivel municipal.

El segundo grupo estaría compuesto por otras 21 políticas de ámbito local y su objetivo básico estaría centrado en la modificación del entorno socioeconómico. 
TABLA A2

POLÍTICAS LOCALES DE MODIFICACIÓN DEL ENTORNO SOCIOECONÓMICO

\begin{tabular}{|c|c|c|}
\hline Política & Variables de respuesta & $\begin{array}{r}\text { Perio- } \\
\text { do de inicio }\end{array}$ \\
\hline Programa Industrialdea & \% de empresas sobre población & 1982 \\
\hline $\begin{array}{l}\text { Memoria de promoción económica } 2006 \text { Vito- } \\
\text { ria -Gasteiz Emprendizaje }\end{array}$ & \% de empresas sobre población & 2006 \\
\hline Seed Capital Bizkaia & $\begin{array}{l}\text { \% de empresas sobre población, } \\
\text { \% de empresas de alta tecnología }\end{array}$ & 1989 \\
\hline Consorcio Transfronterizo Bidasoa-Txingudi & $\begin{array}{l}\text { \% de empresas sobre población, } \\
\% \text { empresas de servicios }\end{array}$ & 1998 \\
\hline Programa De Desarrollo Rural de Gorbea & $\begin{array}{l}\text { \% de empresas sobre población, } \\
\text { \% empresas industriales }\end{array}$ & 2003 \\
\hline Programa Sustatu & \% empresas sobre población & 2007 \\
\hline Programa de Internacionalización de Gipuzkoa & $\begin{array}{l}\text { Inversión exterior, exportacio- } \\
\text { nes }\end{array}$ & 2004 \\
\hline Berritu.biz & $\begin{array}{l}\text { Competitividad, exportaciones } \\
\text { locales, Innovación }\end{array}$ & 2007 \\
\hline Programa Premie & Rentabilidad de las PYMES & 1996 \\
\hline Gipuzkoa Berritzen & Indicadores locales de I+D+i & 2005 \\
\hline $\begin{array}{l}\text { Progaram iGipuzkoa 2010: Udalak / Progaram } \\
\text { iGipuzkoa } 2005\end{array}$ & Indicadores locales de TIC & 2003 \\
\hline Programa i-gipuzkoa 2010 personas & Indicadores locales de TIC & 2003 \\
\hline $\begin{array}{l}\text { Programa municipal de ayudas económicas al } \\
\text { autoempleo femenino }\end{array}$ & $\%$ de autoempleo femenino & 2007 \\
\hline $\begin{array}{l}\text { Txekinbide program de formación para el em- } \\
\text { pleo }\end{array}$ & $\begin{array}{l}\text { Tasas de paro en colectivos ob- } \\
\text { jetivo }\end{array}$ & 2003 \\
\hline Merkataritzan & $\begin{array}{l}\text { Tasas de paro en colectivos ob- } \\
\text { jetivo }\end{array}$ & 2004 \\
\hline Hezilan & $\begin{array}{l}\text { Tasas de paro en colectivos ob- } \\
\text { jetivo }\end{array}$ & 2006 \\
\hline Lanbegi & Tasas de paro, duración del paro & 2006 \\
\hline Plan de Acción 2005-2008 AL21 Bilbao & $\begin{array}{l}\text { \% de energías renovables, Con- } \\
\text { sumo per cápita }\end{array}$ & 2005 \\
\hline Memoria 2003 Turismo Bidasoa & Turismo receptor & 2003 \\
\hline $\begin{array}{l}\text { Programa de Desarrollo Rural de la Comarca } \\
\text { de Cantábrica Alavesa }\end{array}$ & Varios indicadores & 2003 \\
\hline Programa IZARTU & Varios indicadores & 2001 \\
\hline
\end{tabular}

Fuente: Elaboración propia en base al SVDR.

En este grupo de políticas públicas, además de recoger la tipología de las variables de respuesta se ha incluido también, a modo de referencia, el periodo de inicio de los efectos de cada una de las políticas y que marcaría el punto medio de necesidades de información estadística a efectos de aplicación de la metodología que se propone en este trabajo.

En esta ocasión las principales necesidades de información estadística se agruparían en torno a tres grandes bloques: tipología y dinámica empresarial, nuevas tecnologías e I+D+i. Esta necesidad requiere de base de datos local adecuada y homogénea.

Para el caso de las políticas regionales, y del mismo modo que para el caso de las de ámbito local, se identifica un primer grupo de 18 políticas que podrían englobarse dentro de la tipología de acción directa, de la cuales 13 
podrían ser valoradas mediante indicadores de intensidad o uso y 5 de ellas podrían ser objeto de valoración de efectos inducidos sobre el entorno meditante la utilización de variables de respuesta.

TABLA A3

POLÍTICAS REGIONALES DE ACTUACIÓN DIRECTA

\begin{tabular}{|c|c|c|}
\hline Política & $\begin{array}{l}\text { Indicadores de } \\
\text { Intensidad / Uso }\end{array}$ & $\begin{array}{l}\text { Variables de res- } \\
\text { puesta }\end{array}$ \\
\hline \multicolumn{3}{|l|}{$\begin{array}{l}\text { Libro Blanco del Aprendizaje a lo largo de la } \\
\text { vida }\end{array}$} \\
\hline \multicolumn{3}{|l|}{ Plan Vasco de Inmigración } \\
\hline \multicolumn{3}{|l|}{$\begin{array}{l}\text { Catálogo Vasco de Cualificaciones Profesiona- } \\
\text { les }\end{array}$} \\
\hline \multicolumn{3}{|l|}{$\begin{array}{l}\text { Plan de Gestión de Residuos Peligrosos 2003- } \\
2006\end{array}$} \\
\hline \multicolumn{3}{|l|}{$\begin{array}{l}\text { Plan Vasco de Lucha contra el Cambio Climá- } \\
\text { tico }\end{array}$} \\
\hline Observatorio de la Biodiversidad & $\begin{array}{l}\mathrm{N}^{\circ} \text { proyectos, Uso de } \\
\text { la web }\end{array}$ & \\
\hline Listado de Tecnologías Limpias & $\begin{array}{l}\mathrm{N}^{\circ} \text { tecnologías im- } \\
\text { plantadas }\end{array}$ & \\
\hline Observatorio del mercado de trabajo & $\begin{array}{l}\mathrm{N}^{\circ} \text { de estudios reali- } \\
\text { zados }\end{array}$ & \\
\hline CIC-Tourgune & $\begin{array}{l}\mathrm{N}^{\circ} \text { de estudios finan- } \\
\text { ciados }\end{array}$ & \\
\hline Comunidad de Trabajo de los Pirineos & $\begin{array}{l}\mathrm{N}^{\circ} \text { de acciones reali- } \\
\text { zadas }\end{array}$ & \\
\hline $\begin{array}{l}\text { Red de Gobiernos Regionales por el Desarrollo } \\
\text { Sostenible }\end{array}$ & $\begin{array}{l}\mathrm{N}^{\circ} \text { de acciones reali- } \\
\text { zadas }\end{array}$ & \\
\hline Autopista del mar entre Bilbao y Dunkerque & $\begin{array}{l}\text { Volumen de mercan- } \\
\text { cías }\end{array}$ & \\
\hline Programa Tranviario & Usuarios & $\begin{array}{c}\text { Percepción am- } \\
\text { biental Movilidad }\end{array}$ \\
\hline PTS de zonas húmedas & $\begin{array}{l}\mathrm{N}^{\circ} \text { actividades reali- } \\
\text { zadas }\end{array}$ & $\begin{array}{l}\text { Percepción am- } \\
\text { biental. Movilidad }\end{array}$ \\
\hline Plan General de Carreteras 1999-2010 & Km construidos & Movilidad \\
\hline Red Vasca de Ciencia, Tecnología e Innovación & $\begin{array}{l}\mathrm{N}^{\circ} \text { actividades reali- } \\
\text { zadas }\end{array}$ & $\begin{array}{l}\text { Indicadores regio- } \\
\text { nales de } \mathrm{I}+\mathrm{D}+\mathrm{i}\end{array}$ \\
\hline Plataforma Logística Aquitaine-Euskadi & ma Utilización platafor- & $\begin{array}{l}\text { Volumen de co- } \\
\text { mercio }\end{array}$ \\
\hline Plan Director de Transporte Sostenible & $\begin{array}{l}\mathrm{N}^{\circ} \text { actividades reali- } \\
\text { zadas }\end{array}$ & $\mathrm{CO}_{2}{ }^{\text {Niveles de emisión }}$ \\
\hline
\end{tabular}

Fuente: Elaboración propia en base al SVDR.

En este caso, las necesidades de información regional para realizar las correspondientes evaluaciones cuantitativas pasarían por la disponibilidad de indicadores homogéneos entre Comunidades Autónomas de percepción ambiental, movilidad, I+D+i, o emisiones. El resto de políticas públicas de ámbito regional, se incluirían en el grupo de modificación del entorno socioeconómico. 
TABLA A4

POLÍTICAS REGIONALES DE MODIFICACIÓN DEL ENTORNO SOCIOECONÓMICO

\begin{tabular}{|c|c|c|}
\hline Política & Variables de respuesta & $\begin{array}{r}\text { Perio- } \\
\text { do de inicio }\end{array}$ \\
\hline Centros de Investigación Cooperativa & Indicadores regionales de I+D+i & 2006 \\
\hline Gaitek & Indicadores regionales de I+D+i & 2005 \\
\hline Eurobulegoa & Indicadores regionales de $\mathrm{I}+\mathrm{D}+\mathrm{i}$ & 2004 \\
\hline Saiotek & Indicadores regionales de I+D+i & 2002 \\
\hline Programa Ikertu & Indicadores regionales de $\mathrm{I}+\mathrm{D}+\mathrm{i}$ & 2005 \\
\hline Programa Hobeki Digitala & Indicadores regionales TIC & 2007 \\
\hline Konekta Zaitez & Indicadores regionales TIC & 2002 \\
\hline Plan Euskadi en la Sociedad de la Información & Indicadores regionales TIC & 2002 \\
\hline $\begin{array}{l}\text { Programa de ayudas del Ente Vasco de la Ener- } \\
\text { gía (EVE) al desarrollo de las Energías renovables }\end{array}$ & \% de generación con renovables & 2007 \\
\hline $\begin{array}{l}\text { Convenios EVE-Ayuntamientos para la promo- } \\
\text { ción de las energías renovables }\end{array}$ & \% de generación con renovables & 2000 \\
\hline $\begin{array}{l}\text { Acuerdos voluntarios con sectores industria- } \\
\text { les }\end{array}$ & Niveles de emisión $\mathrm{CO}_{2}$ & 2002 \\
\hline Directrices de Ordenación Territorial & Indicadores ambientales & \\
\hline Udalsarea 21 & Indicadores ambientales & 2002 \\
\hline $\begin{array}{l}\text { Orden conjunta del DMAOT para entidades lo- } \\
\text { cales }\end{array}$ & Indicadores ambientales & 2007 \\
\hline Programa Gaztenek & \% empleo agrario por edades & 2004 \\
\hline HOBETUZ & $\begin{array}{l}\text { \% empleo que recibe cursos de } \\
\text { formación }\end{array}$ & 1996 \\
\hline $\begin{array}{l}\text { Plan Director de Seguridad y Salud Laboral } \\
2003-2006\end{array}$ & $\begin{array}{l}\text { Indicadores de siniestralidad la- } \\
\text { boral }\end{array}$ & 2003 \\
\hline Plan Interinstitucional de Empleo 2003-2006 & Tasas de paro & 2003 \\
\hline Lanbide & Tasas de paro & 2005 \\
\hline Fondos de capital riesgo de SPRI & \% de empresas Alta tecnología & 1985 \\
\hline Programa Ekintzaile & Empresas sobre población & 2006 \\
\hline Programa Atzerri & Apertura exterior & 2000 \\
\hline Plan de Desarrollo Rural Sostenible 2007-2013 & $\begin{array}{l}\text { Exportación agraria, \% de pobla- } \\
\text { ción en núcleos rurales. }\end{array}$ & 2005 \\
\hline Programa Erein & $\begin{array}{l}\text { \% de población en núcleos rura- } \\
\text { les }\end{array}$ & 2002 \\
\hline $\begin{array}{l}\text { Plan para la adaptación estructural del sector } \\
\text { de la pesca, la acuicultura y la transformación y co- } \\
\text { mercialización de los productos pesqueros }\end{array}$ & $\begin{array}{l}\text { Productividad en la pesca. Ex- } \\
\text { portaciones }\end{array}$ & 2000 \\
\hline
\end{tabular}

Fuente: Elaboración propia en base al SVDR.

Los indicadores de I+D+i y de nuevas tecnologías serían los más demandados para estas aplicaciones, seguidos de indicadores medioambientales y de formación y empleo. 


\section{ANEXO 2}

\section{EJES BÁSICOS Y LÍNEAS DE ACTUACIÓN DEL PROGRAMA IZARTU}

Para alcanzar estos objetivos, el programa se estructura en torno a 5 ejes básicos, cada uno de los cuales incorpora, a su vez distintas líneas de actuación, tal como se recoge en el cuadro que presentamos a continuación.

Ejes básicos y líneas de actuación del Programa Izartu.

\begin{tabular}{|c|c|}
\hline Ejes & Líneas de actuación \\
\hline \multirow{4}{*}{$\begin{array}{l}\text { 1.-Entorno productivo y } \\
\text { empleo }\end{array}$} & 1.1. Creación y desarrollo de estructuras de apoyo a la actividad \\
\hline & 1.2. Creación y desarrollo de infraestructura productiva. \\
\hline & 1.3. Apoyo al empresariado, PYMES y empresas artesanales. \\
\hline & 1.4. Promoción local, turismo y patrimonio \\
\hline \multirow{8}{*}{$\begin{array}{l}\text { 2.- Infraestructuras básicas } \\
\text { locales }\end{array}$} & 2.1. Infraestructuras de transporte. \\
\hline & $\begin{array}{l}\text { 2.2. Infraestructura Telecomunicaciones y Sociedad Informa- } \\
\text { ción }\end{array}$ \\
\hline & 2.3. Infraestructura energética. \\
\hline & 2.4. Infraestructura medioambiental \\
\hline & 2.5. Infraestructuras Ordenación y Rehabilitación. \\
\hline & 2.6. Infraestructura Social y Sanitaria. \\
\hline & 2.7. Infraestructura Ocio y Cultura. \\
\hline & 2.8 Infraestructura Educativa y Formativa. \\
\hline \multirow{4}{*}{$\begin{array}{l}\text { 3.-Recursos humanos e in- } \\
\text { tegración social }\end{array}$} & 3.1. Políticas del mercado laboral. \\
\hline & 3.2. Integración social. \\
\hline & 3.3. Igualdad de oportunidades. \\
\hline & 3.4. Educación y formación. \\
\hline \multirow{3}{*}{$\begin{array}{l}\text { 4.- Creación y desarrollo de } \\
\text { estructuras básicas locales }\end{array}$} & 4.1. Servicios Sociales básicos. \\
\hline & 4.2. Seguridad ciudadana. \\
\hline & 4.3. Educación y formación cívica. \\
\hline 5.- Otras & 5.1. Asistencia Técnica. \\
\hline
\end{tabular}

Hasta el año 2003 se habían presentado un total de 350 proyectos, de los que una gran parte $73 \%$ se enmarcaban dentro eje 2 (255 proyectos), siendo las infraestructuras de Ordenación y Rehabilitación las más demandas con 151 proyectos.

En un segundo nivel de demanda no encontramos con los ejes 1 ( 33 proyectos) y 3 ( 31 proyectos), suponiendo cada uno de ellos algo menos del 10\% del total de proyectos presentados. Finalmente, el eje 5 absorbió el $7 \%$ de los proyectos (23) y el eje 4 el $2 \%$ ( 8 proyectos). 